\title{
Planetary transit candidates in the CoRoT LRa01 field ${ }^{\star}$
}

\author{
L. Carone ${ }^{1}$, D. Gandolfi' ${ }^{2,3}$, J. Cabrera ${ }^{4,5}$, A. P. Hatzes ${ }^{3}$, H. J. Deeg ${ }^{6,7}$, Sz. Csizmadia ${ }^{4}$, M. Pätzold ${ }^{1}$, J. Weingrill ${ }^{8}$,
} S. Aigrain ${ }^{9}$, R. Alonso ${ }^{10}$, A. Alapini ${ }^{11}$, J.-M. Almenara ${ }^{6,7,12}$, M. Auvergne ${ }^{13}$, A. Baglin ${ }^{13}$, P. Barge ${ }^{12}$, A. S. Bonomo ${ }^{12}$, P. Bordé ${ }^{14}$, F. Bouchy ${ }^{15,16}$, H. Bruntt ${ }^{13}$, S. Carpano ${ }^{2}$, W. D. Cochran ${ }^{17}$, M. Deleuil ${ }^{12}$, R. F. Díaz ${ }^{15,16}$, S. Dreizler ${ }^{18}$, R. Dvorak ${ }^{19}$, J. Eislöffel ${ }^{3}$, P. Eigmüller ${ }^{3}$, M. Endl ${ }^{17}$, A. Erikson ${ }^{4}$, S. Ferraz-Mello ${ }^{20}$, M. Fridlund ${ }^{2}$, J.-C. Gazzano ${ }^{12,21}$, N. Gibson ${ }^{9,11}$, M. Gillon ${ }^{10,22}$, P. Gondoin ${ }^{2}$, S. Grziwa ${ }^{1}$, E. W. Günther ${ }^{3}$, T. Guillot ${ }^{21}$, M. Hartmann ${ }^{3}$, M. Havel ${ }^{21}$, G. Hébrard ${ }^{15}$, L. Jorda ${ }^{12}$, P. Kabath ${ }^{4,23}$, A. Léger ${ }^{14}$, A. Llebaria ${ }^{12}$, H. Lammer $^{8}$, C. Lovis ${ }^{10}$, P. J. MacQueen ${ }^{17}$, M. Mayor ${ }^{10}$, T. Mazeh ${ }^{24}$, C. Moutou ${ }^{12}$, L. Nortmann ${ }^{18}$, A. Ofir ${ }^{24}$, M. Ollivier ${ }^{14}$, H. Parviainen ${ }^{6,7}$, F. Pepe ${ }^{10}$, F. Pont ${ }^{11}$, D. Queloz ${ }^{10}$, M. Rabus ${ }^{6,7,25}$, H. Rauer, ${ }^{4,26}$, C. Régulo ${ }^{6,7}$, S. Renner ${ }^{4,27,28}$, R. de la Reza ${ }^{29}$, D. Rouan ${ }^{13}$, A. Santerne ${ }^{12}$, B. Samuel ${ }^{14}$, J. Schneider ${ }^{5}$, A. Shporer ${ }^{24,30}$, B. Stecklum ${ }^{3}$, L. Tal-Or ${ }^{24}$, B. Tingley ${ }^{6,7}$, S. Udry ${ }^{10}$, and G. Wuchterl ${ }^{3}$

(Affiliations can be found after the references)

Received 28 March 2011 / Accepted 10 October 2011

\section{ABSTRACT}

Context. CoRoT is a pioneering space mission whose primary goals are stellar seismology and extrasolar planets search. Its surveys of large stellar fields generate numerous planetary candidates whose lightcurves have transit-like features. An extensive analytical and observational follow-up effort is undertaken to classify these candidates.

Aims. We present the list of planetary transit candidates from the CoRoT LRa01 star field in the Monoceros constellation toward the Galactic anti-center direction. The CoRoT observations of LRa01 lasted from 24 October 2007 to 3 March 2008.

Methods. We acquired and analyzed 7470 chromatic and 3938 monochromatic lightcurves. Instrumental noise and stellar variability were treated with several filtering tools by different teams from the CoRoT community. Different transit search algorithms were applied to the lightcurves.

Results. Fifty-one stars were classified as planetary transit candidates in LRa01. Thirty-seven (i.e., 73\% of all candidates) are "good" planetary candidates based on photometric analysis only. Thirty-two (i.e., $87 \%$ of the "good" candidates) have been followed-up. At the time of writing twenty-two cases were solved and five planets were discovered: three transiting hot-Jupiters (CoRoT-5b, CoRoT-12b, and CoRoT-21b), the first terrestrial transiting planet (CoRoT-7b), and another planet in the same system (CoRoT-7c, detected by radial velocity survey only). Evidence of another non-transiting planet in the CoRoT-7 system, namely CoRoT-7d, was recently found as well.

Key words. techniques: spectroscopic - techniques: photometric - binaries: eclipsing - techniques: radial velocities planetary systems

\section{Introduction}

We here summarize the planetary candidates found in the LRa01 exoplanet star field and some preliminary scientific results from the combination of CoRoT photometry with groundbased follow-up observations. The CoRoT IRa01 and LRc01 runs have already been reported by Carpano et al. (2009) and Moutou et al. (2009), and Cabrera et al. (2009), respectively.

The LRa01 run, from 24 October 2007 to 3 March 2008, was the second long pointing of CoRoT after the LRc01 field (Cabrera et al. 2009). The LRa01 star field contains 11408 pre-selected stars covering a sky-area within the coordinates $06^{\mathrm{h}} 45^{\mathrm{m}} 11.2^{\mathrm{s}} \leq \mathrm{RA} \leq 06^{\mathrm{h}} 45^{\mathrm{m}} 59.4^{\mathrm{s}}$ and $-01^{\circ} 27^{\prime} 21^{\prime \prime} \leq$ $\delta \leq+01^{\circ} 6^{\prime} 23^{\prime \prime}$ (J2000) in the Monoceros constellation, toward the Galactic anti-center direction.

Fifty-one transit candidates have been identified in LRa01 (Tables 5 and 6). Four transiting planets have been discovered and confirmed: CoRoT-5b (Rauer et al. 2009), CoRoT-12b (Gillon et al. 2010), and CoRoT-21b (Pätzold et al., in prep.), three Jupiter-size planets with $M_{\mathrm{p}}=0.47,0.92$, and $\approx 2 M_{\text {Jup }}$,

* The CoRoT space mission, launched on December 27, 2006, has been developed and is operated by CNES, with contributions of Austria, Belgium, Brazil, ESA (RSSD and Science Program), Germany and Spain. respectively; CoRoT-7b, the first terrestrial transiting planet (Léger et al. 2009; Queloz et al. 2009). A non-transiting planet with a mass of about $8 M_{\text {Earth }}$ in the CoRoT-7 system, namely CoRoT-7c, was also detected by radial velocity (RV) observations only (Queloz et al. 2009). The potential discovery of a third planet in the CoRoT-7 system, CoRoT-7d, was reported by Hatzes et al. (2010). A list of photometrically identified eclipsing binary systems is presented in Table 7 . Identified variable stars of the first four CoRoT exoplanet star fields are reported in Debosscher et al. (2009).

We report on the characteristics of the LRa01 star-field (Sect. 2), the CoRoT photometry and nature of different instrumental systematic effects (Sect. 3), the transit detection (Sect. 4), and the observing strategy of ground-based follow-ups (Sect. 5). The process of resolving the nature of CoRoT candidates is described in Moutou et al. (2009). A description of all detected transit candidates is presented in Sect. 6. Results are discussed in terms of detection efficiency compared to previous CoRoT runs (Sect. 7). We give a summary in Sect. 8.

\section{Field characterization}

During the CoRoT mission preparatory phase, a massive and deep Harris $B V$ and Sloan $r^{\prime} i^{\prime}$ photometric survey was 


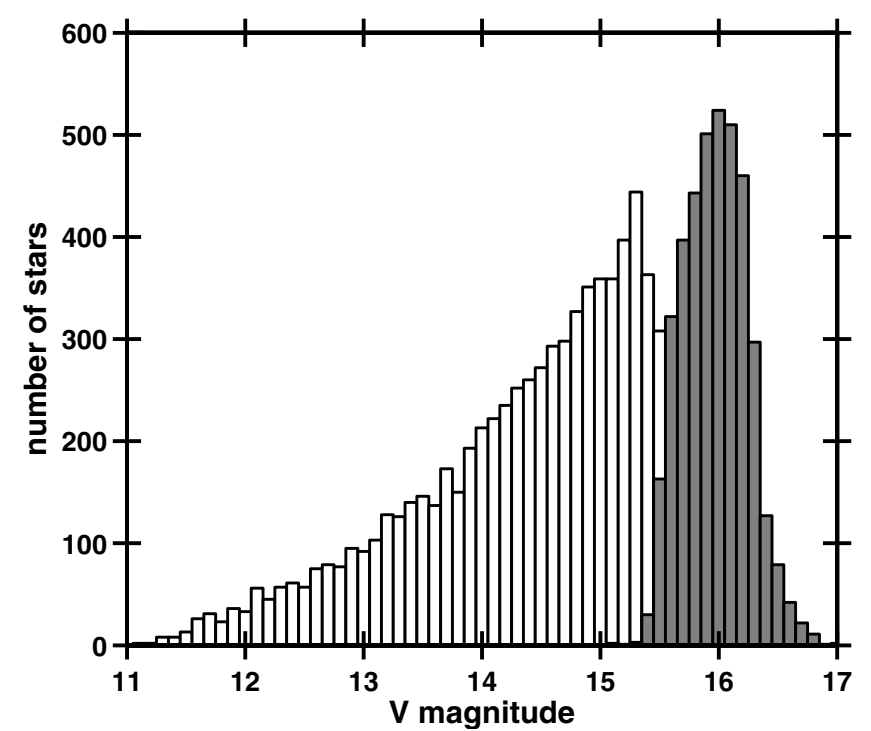

Fig. 1. Histogram of the the visual magnitudes of the stars observed by CoRoT in the LRa01 exoplanet star field. Gray: monochromatic lightcurves. White: chromatic lightcurves. The majority of the targets are relatively faint stars with $V>14$ mag.

performed in the CoRoT exoplanet fields using the Wide Field Camera (WFC) at the Isaac Newton Telescope (INT). The goals were i) to perform a first-order spectral classification of the stars in the fields; ii) to determine their position with sufficient accuracy for a precise placement of the CoRoT photometric masks; and iii) to assess the level of contamination from background/foreground objects within a few arc-seconds from the CoRoT target stars. The relevant information is collected in the Exo-Dat database ${ }^{1}$ (Deleuil et al. 2006, 2009; Meunier et al. 2007).

CoRoT was designed to fulfill two main objectives: conducting stellar seismology studies of interesting stars and searching for extrasolar planets. Astroseismology requires high signal-tonoise $(S / N)$ ratio photometry and it is thus focused mainly on the study of relatively bright targets: typically $\sim 10$ stars with $V<$ 9.5 mag are observed in each CoRoT seismo-field. On the other hand, the transiting exoplanet search requires a large number of targets because of the low probability to find planets whose orbits are oriented such that transits can be observed in front of their host stars (the probability is about $5 \%$ for semi-major axes of $0.1 \mathrm{AU}$ ). The selection of the observed CoRoT seismoand $e x o$-fields thus represents a compromise between these two requirements.

We observed 11408 stars with visual magnitudes $11 \lesssim V \lesssim$ 17 mag for the transit search in the LRa01 field (Fig. 1). Dwarf stars are optimal targets for the photometric search of extrasolar planets (Michel et al. 2008; Gondoin et al. 2009; Hekker et al. 2009). Therefore, the percentage of Sun-like stars in a field is important to estimate the detection efficiency. We estimated the percentage of giant and dwarf stars in LRa01 by combining the Exo-Dat optical photometry (Deleuil et al. 2009) with the nearinfrared Two Micron All Sky Survey (2MASS) Point Source catalog $^{2}$ (Cutri et al. 2003) and using color-magnitude diagrams (Fig. 2). Although it is difficult to find a clear distinction between

\footnotetext{
${ }^{1}$ http://lamwws.oamp.fr/exodat/

2 The near-infrared JHKs 2MASS catalog is available at http://irsa.ipac. caltech.edu/applications/2MASS/IM/ interactive.html
}
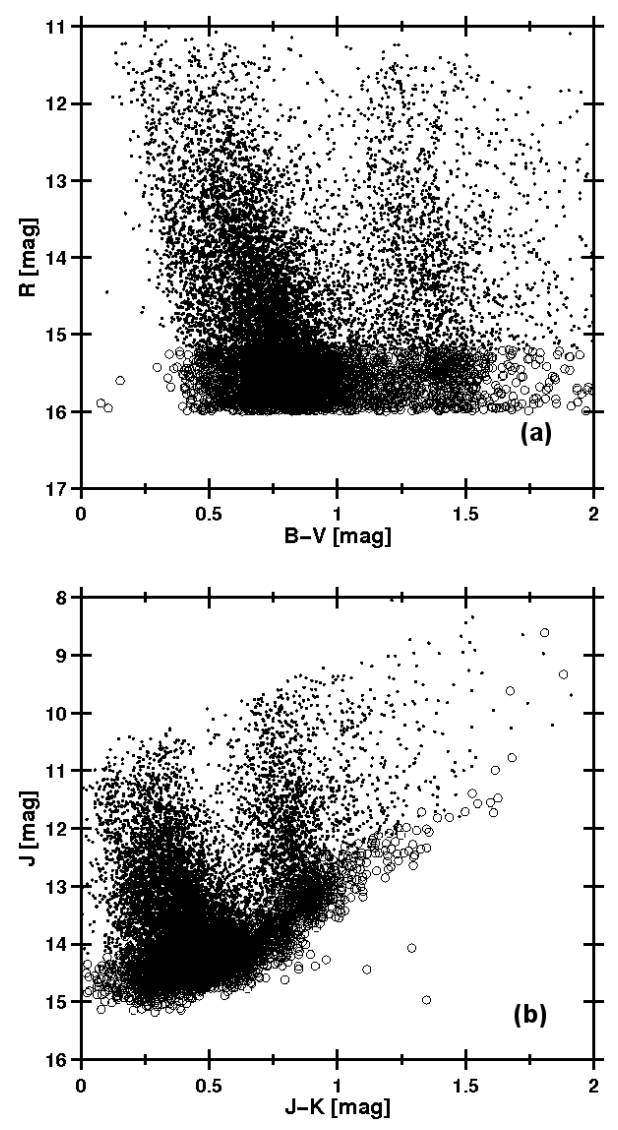

Fig. 2. Top panel: $r^{\prime}$ versus $B-V$ color-magnitude diagram of the stars in the CoRoT LRa01 star field. The dots mark the bright stars $\left(r^{\prime} \lesssim 15.2 \mathrm{mag}\right.$ ), for which three-color CoRoT lightcurves are available. The open circles mark the fainter stars $\left(r^{\prime} \gtrsim 15.2 \mathrm{mag}\right)$ with monochromatic lightcurves only. Main-sequence stars cluster in the left part of the diagram, giant stars in the right part. Bottom panel: 2MASS $J$ versus $J-K$ s color-magnitude diagram of the stars in the CoRoT LRa01 field. Again, main-sequence stars cluster in the left part of the diagram, giant stars in the right part.

these two populations using broad-band photometry (in particular for stars with $V \gtrsim 15 \mathrm{mag}$ ), $75 \%$ of the stars observed by CoRoT in LRa01 seem to be dwarf stars (Fig. 2). The distribution of luminosity classes derived from Exo-Dat only shows a similar picture (Fig. 3): $\sim 0.50 \%$ of the stars in the LRa01 exoplanet field are supergiants, $\sim 3 \%$ are bright giants, $\sim 15 \%$ are giants, $\sim 31.5 \%$ are subgiants, and $\sim 50 \%$ are dwarfs. This is of advantage for the search of extrasolar planets compared to the LRc01 star field, where only $\sim 37.3 \%$ of the stars are dwarfs (Cabrera et al. 2009). However, this analysis is valid from a statistical point of view. Photometric criteria can indeed lead to misclassification of individual stars (see Klement et al. 2011, and reference therein). The Exo-Dat spectral classification, which is based only on broad-band photometry, suffers some uncertainties, the main ones being the star reddening, the unknown chemical abundances, and the potential binarity, which can result in a wrong identification of the spectral and luminosity classes of the stars. Based on multi-object, intermediate-resolution spectroscopy performed with VLT/FLAMES on a subset of stars in LRa01, Gazzano et al. (2010) found that the photometrically classified dwarf content is underestimated by about $15 \%$. 


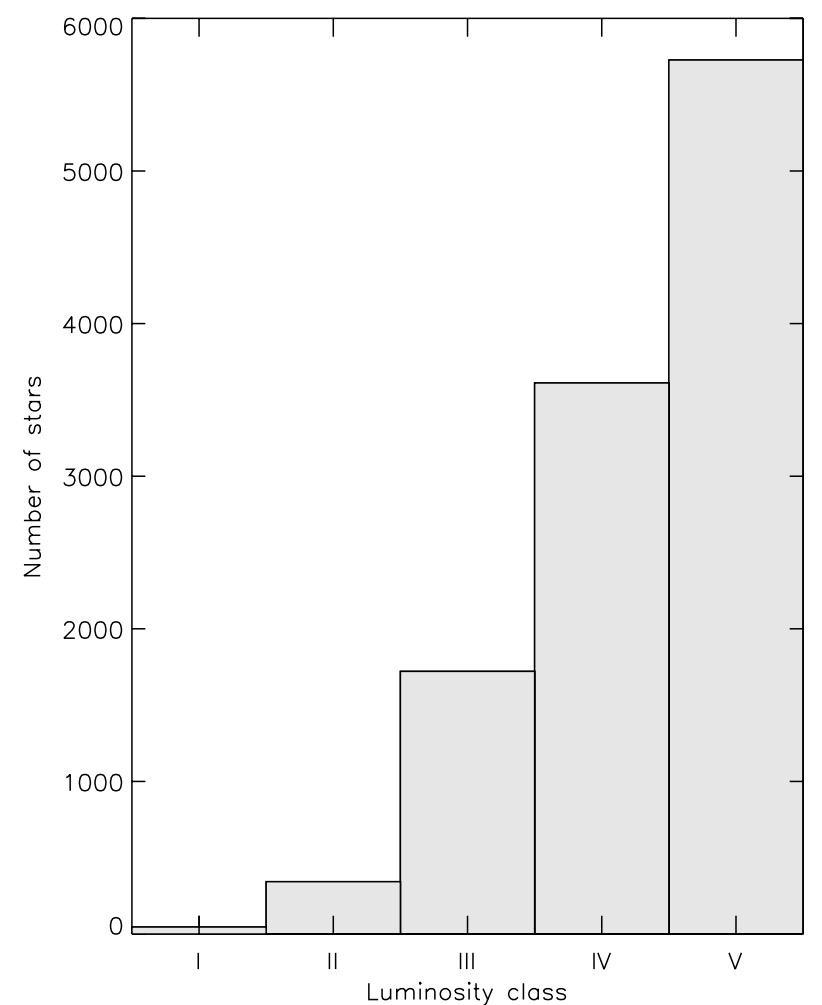

Fig. 3. Histogram of the luminosity classes of the LRa01 star field as derived from Exo-Dat (Deleuil et al. 2009). The majority of the target stars $(\sim 50 \%)$ are main-sequence dwarf stars (luminosity class $\mathrm{V}$ ). Subgiant (luminosity class IV), giant (luminosity class III), bright giant (luminosity class II), and supergiant stars (luminosity class I) make up $\sim 31.5 \%, \sim 15 \%, \sim 3 \%$, and $\sim 0.50 \%$ of the total, respectively.

\section{CoRoT photometry, data reduction, and systematic effect}

CoRoT data are made public after a proprietary period of one year. The data from LRa01 were released to the CoIs on 29 October 2008 and to the public on 29 October 2009. CoRoT lightcurves are identified by either the CoRoT ID, a unique 10-digit number, or the so-called CoRoT "Win ID" (i.e., window ID). The "Win ID" contains the identifier E1 or E2, which identifies the CCD 1 or 2 of the exoplanet channel, respectively, followed by a 4-digit number. This number represents the assigned CoRoT mask. The "Win ID" is re-used for every run. To identify an individual lightcurve by the "Win ID", the data acquisition run is required. In addition, a threecharacter abbreviation, CHR or MON, is given for the identification of chromatic or monochromatic lightcurves, respectively. For example, the lightcurve of the star CoRoT-7, which was observed during LRa01 and harbours the first transiting terrestrial planet (Léger et al. 2009), is labeled by the following identification: LRa01 E2 0165 - CHR - 0102708694.

A bi-prism was installed in the exoplanet channel to disperse the flux of the observed stars. Three-color photometry is obtained by splitting the point-spread function into three subareas based on the dispersion property of the bi-prism (blue light is stronger dispersed than red light) for the targets with visual magnitudes $V \lesssim 15.2 \mathrm{mag}$, i.e., about $65 \%$ of the lightcurves in this run. Flux from these areas is defined as red, green, and blue. The CoRoT color channels, however, do not correspond to any standard photometric systems (Auvergne et al. 2009).

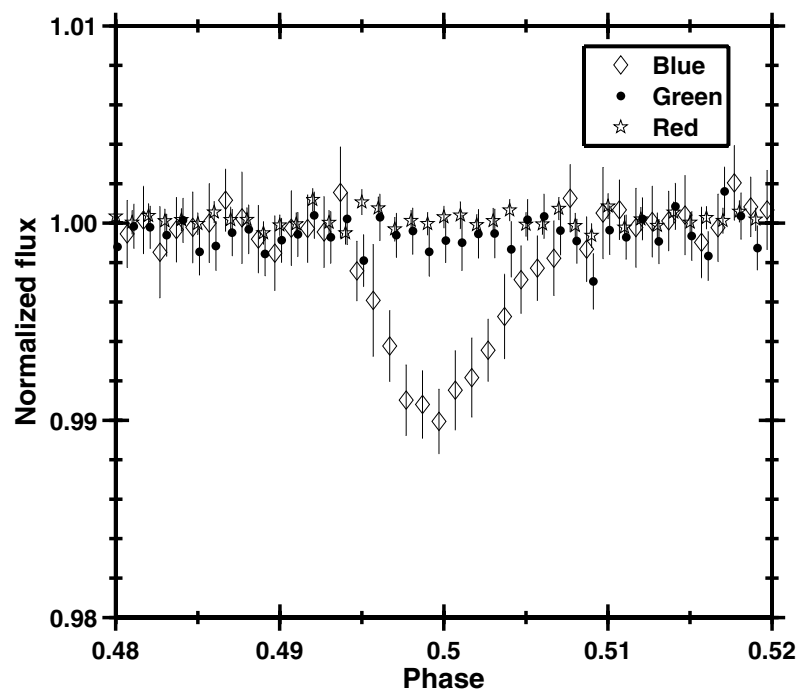

Fig. 4. Phase-plot of the candidate LRa01 E2 2597 (CoRoT ID 0102672065) folded at the period $P=8.90$ days in the threecolor channels red (star), green (circle), and blue (diamond). The lightcurves are normalized to the fluxes in the respective colors. The event is only visible in the blue channel. This indicates a contamination from a background binary system.

The chromatic information is helpful to distinguish between achromatic planetary transits and chromatic eclipsing binaries. The chromatic information is also used to identify false alarms from diluted background binaries. After an accurate study of the light contamination inside the photometric mask, candidates with 3 sigma significant depth differences in the three CoRoT channels are usually flagged as potential contaminating eclipsing binaries. As an example, the CoRoT target LRa01 E2 2597 has a deep transit in the blue channel ${ }^{3}$, which is not detected in the green and red channels at a $12 \sigma$ and $25 \sigma$ significance, respectively (Fig. 4). If the signal were on target, the "green" and "red" transit signals would have been clearly detected. As a consequence, this candidate is identified as a contaminating eclipsing binary (CEB). In other cases, i.e., LRa01 E1 2101 (Sect. 6.3.2) and LRa01 E2 3156 (Sect. 6.3.13), the signal is detected in only one channel but the expected signal strengths in the other channels are below the noise threshold. Therefore, we could not exclude these signals as arising from a contaminating eclipsing binary. Indeed, ground-based photometric follow-up concluded that the transit signal of LRa01 E1 2101 and LRa01 E2 3156 are likely on target.

Several systematic effects need to be filtered out during the data reduction to achieve maximum accuracy. The main perturbation factors are eclipses (when the spacecraft enters the shadow of the Earth), which cause short-term temperature fluctuations on the spacecraft, the Earth's gravity and magnetic field, solar and terrestrial infrared emissivity, the Earth's albedo, and objects in low-Earth orbit. Known instrumental effects like spacecraft jitter are already removed in the processing. A detailed description of lightcurve perturbations and employed corrections is given in Auvergne et al. (2009), and Drummond et al. (2008) and Pinheiro da Silva et al. (2008), respectively. Details about onboard data-reduction can be found in Llebaria \& Guterman (2006).

\footnotetext{
${ }^{3}$ One percent deep when normalized to the blue flux only, $0.14 \%$ deep when normalized to the total flux of all three channels.
} 


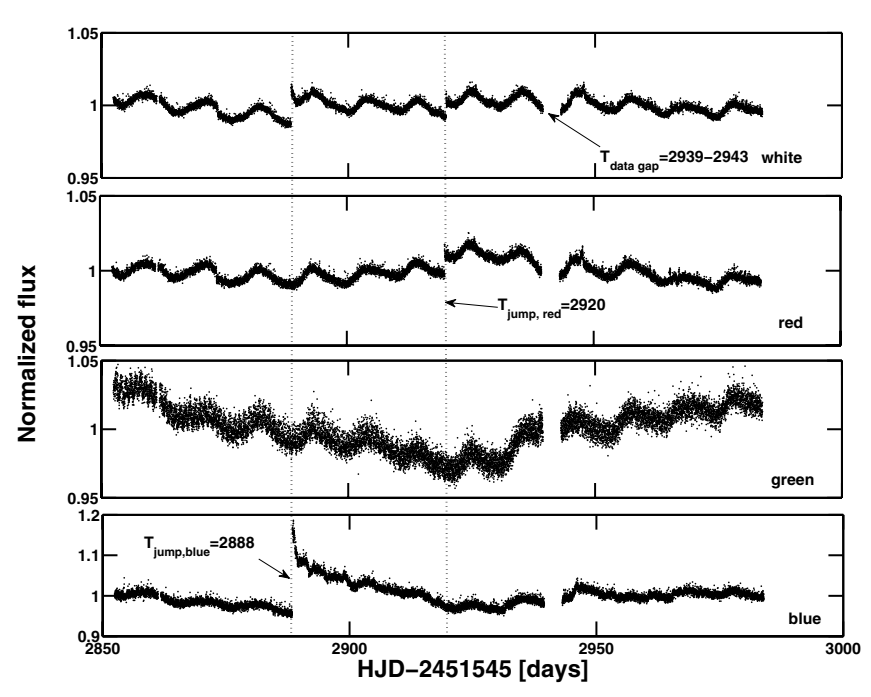

Fig. 5. Chromatic lightcurves of the star LRa01E1 2698. From top to bottom: white (red + green + blue), red, green, and blue lightcurve versus observing time in HJD. The flux of each lightcurve is normalized to 1 . The red channel contributes the most to the combined white lightcurve (79\% of the flux). Ten percent of the combined white flux originates from the green channel, whereas the blue channel contributes $11 \%$ of the total white flux. The dashed lines indicate two "hot-pixel" events occurred in the blue and red channels.

Irradiation excites single pixels, which are difficult to correct. These "hot-pixels" appear predominantly in orbit when crossing the south Atlantic anomaly (SAA). A value in the header of each lightcurve informs the user how many "hotpixels" were identified in the lightcurve. Not all "hot-pixels" are identified during the data processing. Unfortunately, these events may mimic a transit-like signal. If only one pixel is affected, this can be identified by comparing the flux in the different color channels (if available).

Although the processing pipeline significantly reduces the noise and removes the systematics, some instrumental effects still remain. As an example, the three color-channel lightcurves of the star LRa01 E1 2698 (CoRoT ID 0102566329), are plotted in Fig. 5, along with the white lightcurve (red + green + blue). Instrumental signatures significantly perturb the lightcurves. On 2454433 HJD ( $T_{\text {jump,blue }}=2888$ days in Fig. 5) a strong "hot-pixel" appeared in the blue channel. It perturbed the white lightcurve only weakly since the flux contribution from the blue channel is small. On 2454465 HJD ( $T_{\text {jump,red }}=2920$ days in Fig. 5) a small "hot-pixel" appeared in the red channel. The effect is seen in the white lightcurve with almost the same amplitude because the red channel contributes most to the overall flux. It should also be noted that the relaxation time for this "hotpixel" is very short compared to the strong event in the blue channel. The flux after the incident settles on a slightly higher level than before. This example proves the usefulness of chromatic data to identify "hot-pixels".

There is a data gap of 3.68 days between HJD 2454484 and $2454488\left(T_{\text {data gap }}=2939-2943\right.$ days in Fig. 5). A proton impact led to a reset of the data processing unit (DPU) 1, which is responsible for data collection on the E1 CCD, on 18 January 2008 at 22:45:57 UT during the SAA crossing. The recording resumed on 22 January 2008 at 14:37:41 UT. All lightcurves in LRa01 originating from CCD 1 contain this data gap.
In order to correct for the effects not removed by the CoRoT pipeline, each detection team inside the CoRoT exoplanet team uses a set of additional filters before applying the transit search algorithms. A description of the various methods applied by each detection team to analyze the CoRoT lightcurves is given in Alapini \& Aigrain (2008), Bordé et al. (2007), Carpano \& Fridlund (2008), Grziwa et al. (in prep.), Moutou et al. (2005, 2007), Régulo et al. (2007), and Renner et al. (2008).

\section{Detection of CoRoT candidates}

The transit candidate selection for each run is performed twice: by the "alarm mode" during the onboard observation, and by the CoRoT detection teams after completion of the onboard observation and the full reduction of the CoRoT data.

The "alarm mode" checks for planetary transits every two weeks when the CoRoT observations are still ongoing (Surace et al. 2008). If the "alarm mode" detects a promising candidate with a planetary transit-like event, the sampling for this specific lightcurve is increased from 512 to $32 \mathrm{~s}$. A preliminary candidate list is created and the candidate coordinates, along with estimated transit properties, are forwarded to the followup teams who perform the ground-based observation campaign (Sect. 5). A dedicated analysis and transit search is performed when the processed data are distributed to the different detection teams within the CoRoT exoplanet science team (typically several months after the end of the CoRoT observation).

The detection teams benefit from the total temporal length of the lightcurves (about 130 days for LRa01) and the reduced noise after full processing, and are well equipped for finding shallow transits around highly variable and active stars. The detection teams try to sort out as many false positives as possible to avoid the waste of observation time and telescope resources on non-planetary objects. See Cabrera et al. (2009) for a full description of the CoRoT detection pipeline, with the generation of an initial candidate list and subsequent vetting for the presence of false alarms arising from eclipsing binaries. In the end, a prioritized list of planet candidates and their parameters is composed, which is subject to photometric and spectroscopic follow-up observations (Sect. 5).

The list of the LRa01 candidates is given in Table 5, along with coordinates, transit period, epoch, duration and depth. Each candidate is described in detail in Sect. 6.

\section{Ground-based follow-up observations of the CoRoT candidates}

The detection of planetary transit candidates in the CoRoT lightcurves is only the start on a long and time-consuming road to the assessment of their real nature. The CoRoT follow-up is performed with ground-based facilities, following the strategy outlined in Alonso et al. (2004). Ground-based follow-up observations are motivated by the need to exclude false positives from the list of candidates and obtain full characterizations of the detected planets. Practically all false positives in transit searches are caused by some configuration involving eclipsing binary systems, with the large majority of false positives caused by either grazing or diluted eclipsing binaries (Sect. 5.2). Their nature was first described by Brown (2003), with a development for CoRoT candidates given by Almenara et al. (2009).

Photometric observations are needed to confirm that the transit is occurring "on target" (Sect. 5.1). If confirmed, complementary RV observations are performed to definitely assess the 
Table 1. Instruments used for the ground-based CoRoT follow-up observations.

\begin{tabular}{|c|c|}
\hline Instrument & Observatory \\
\hline \multicolumn{2}{|c|}{ Photometry } \\
\hline $\begin{array}{l}\text { CAMELOT on the IAC } 80 \mathrm{~cm} \text { telescope; SD camera on the } \\
\text { ESA Optical ground-station (ESA-OGS); FastCam at the } \\
\text { Carlos Sanchez Telescope (CST) }\end{array}$ & Observatorio del Teide, Tenerife, Spain \\
\hline CCD cameras on the $0.46 \mathrm{~m}$ and $1 \mathrm{~m}$ Wise telescopes & Wise Observatory, Israel \\
\hline EulerCam on the Euler $1.2 \mathrm{~m}$ telescope & La Silla observatory, Chile \\
\hline $\begin{array}{l}\text { MegaCam on the } 3.6 \mathrm{~m} \text { Canada-France-Hawaii telescope } \\
\text { (CFHT) }\end{array}$ & CFH observatory, Hawaii, USA \\
\hline CCD camera on the $1.2 \mathrm{~m}$ MONET-North telescope & McDonald Observatory, Texas, USA \\
\hline NACO on the ESO-VLT & Paranal Observatory, Chile \\
\hline BEST on the DLR $20 \mathrm{~cm}$ telescope & Observatoire de Haute-Provence (OHP), France \\
\hline BEST II on the DLR $25 \mathrm{~cm}$ telescope & Observatorio Cerro Armazones, Chile \\
\hline \multicolumn{2}{|c|}{ Spectroscopy } \\
\hline AAOmega on the $3.9 \mathrm{~m}$ AAO telescope & Astronomical Australian Observatory (AAO), Australia \\
\hline FLAMES-GIRAFFE and UVES on ESO's VLT & Paranal Observatory, Chile \\
\hline CRIRES on ESO's VLT & Paranal Observatory, Chile \\
\hline $\begin{array}{l}\text { CORALIE on the } 1.2 \mathrm{~m} \text { Euler telescope, HARPS on the ESO } \\
3.6 \mathrm{~m} \text { telescope }\end{array}$ & La Silla observatory, Chile \\
\hline Sandiford on the $2.1 \mathrm{~m}$ Otto Struve telescope & McDonald Observatory, Texas, USA \\
\hline Coudé spectrograph on the $2 \mathrm{~m}$ TLS telescope & Thüringer Landessternwarte (TLS), Tautenburg, Germany \\
\hline SOPHIE on the $1.93 \mathrm{~m}$ telescope & Observatoire de Haute-Provence (OHP), France \\
\hline HIRES on the $10 \mathrm{~m}$ Keck I telescope & Keck observatory, Hawaii,USA \\
\hline FIES on the $2.56 \mathrm{~m}$ NOT telescope & Roque de los Muchachos Observatory, La Palma, Spain \\
\hline
\end{tabular}

planetary nature of the transiting body and eventually reveal the true mass of the planet. High $S / N$ ratio spectroscopy at highresolution is also conducted to derive the photospheric fundamental parameters of the planet host star and determine its mass and radius (Sect. 5.2).

The CoRoT follow-up program is challenging both in terms of telescope capabilities and observation time. The CoRoT follow-up community uses time allocated at different observatories as described in Sects. 5.1 and 5.2. Many RV observations in 2008 and 2009 were dedicated to CoRoT-7 to confirm the existence and nature of the companions and to constrain their parameters (Léger et al. 2009; Queloz et al. 2009; Hatzes et al. 2010, 2011). Follow-up observations for other candidates were therefore limited or delayed to the 2009/2010 and 2010/2011 observing season. Table 1 lists all facilities used for the follow-up of the LRa01 candidates, Table 6 lists the results of the followup in a concise form. Details can be found in the description of the individual candidates in Sect. 6.

\subsection{Photometry}

The first step in the follow-up sequence is the ground-based photometric observation of the transit. It needs to be verified that the detected transit signals occur on the main target inside the CoRoT photometric mask (typically 20" large). The star is observed during a transit (on observation), and again between two transits (off observation). The stellar brightness, as well as that of any other nearby star is monitored. Contaminating eclipsing binaries (CEBs) are sorted out by this procedure. The on-off photometric method is described in more detail in Deeg et al. (2009).

Required for these observations is the correct and precise prediction of the epoch of the transit occurrence. Timing errors of more than a few hours make the follow-up of transit events unfeasible. The ephemeris errors of faint or shallow candidates (listed in Table 5) imply that their follow-up has to be performed within 1-2 years after CoRoT onboard observation.

As part of the photometric follow-up program of CoRoT, the BEST telescope at OHP performed a survey of variable stars in the LRa01 field prior to the satellite launch (Kabath et al. 2008). The eclipsing binaries LRa01 E1 1574 and LRa01 E1 0622 (Table 7) are among the targets previously found in the aforementioned survey.

\subsection{Spectroscopy}

The conclusion on the nature of some transiting objects is drawn from complementary time-series RV measurements as well as high-resolution, high $S / N$ ratio spectroscopy.

Radial velocity measurements are required to reject possible false-positives and confirm the planetary nature of the transiting object. Binary systems, when they are eventually identified by RV measurements, are classified into the following categories: i) binaries with only one spectroscopically visible stellar component (SB1); ii) binaries with two or more spectroscopically visible stellar components (SB2, SB3, etc.); iii) blended eclipsing binaries, i.e., spatially unresolved eclipsing binaries whose light is diluted by the main CoRoT target (blend scenario).

Transits provide the direct measurement of the planet-to-star radius ratio $\left(R_{\mathrm{P}} / R_{*}\right)$, whereas $\mathrm{RV}$ measurements yield the mass function of the star/planet system. Stellar radii and masses are therefore needed to determine radii and masses of the transiting candidates. A first-order estimate of the size of the transiting objects is derived from the spectral types of the host-stars, as listed in the Exo-Dat data-base. However, as already described in Sect. 2, the photometrically spectral classification reported in Exo-Dat suffers from some uncertainties. High-resolution, high $S / N$ ratio spectroscopy is consequently necessary to derive stellar masses and radii and, eventually, determine the mass, radius, and bulk density of the confirmed planets.

The spectroscopic follow-up observations of LRa01 started with a first spectroscopic "snap-shot" of some of the CoRoT candidates. Low-resolution $(R \approx 1300)$ reconnaissance spectroscopy was performed with the AAOmega multi-fiber spectrograph mounted at the 3.9 m telescope of the Australian Astronomical Observatory (AAO) during two observing runs, 
in January 2008 and from December 2008 to January 2009 (Günther et al. 2012; Sebastian et al. 2012). Further multi-object spectroscopic observations were performed with the FLAMESGIRAFFE facility $(R \approx 26000)$ at the ESO Very Large Telescope (Paranal Observatory, Chile) in January 2005 (Loeillet et al. 2008; Gazzano et al. 2010).

The AAOmega and FLAMES-GIRAFFE observations classified the stars and derived a fist estimate of their photospheric parameters as described in Gandolfi et al. (2008) and Gazzano et al. (2010). If the host star turned out to be a giant star although it was listed as a main-sequence star in the Exo-Dat database, the size of the transiting object was re-evaluated based on the new stellar parameters. Planned ground-based photometry and RV follow-up observations were cancelled if the size of the transiting body was inconsistent with a planetary object. This spectroscopic screening singled out also B-type stars and rapidly rotating targets for which high-precision RV measurements are not feasible.

The nature of the transiting objects is further investigated through reconnaissance high-resolution spectroscopy. This is performed using the CORALIE spectrograph at the $1.2 \mathrm{~m}$ Euler telescope in La Silla observatory, the Sandiford cassegrain echelle spectrograph on the $2.1 \mathrm{~m}$ telescope at McDonald Observatory, and the coudé echelle spectrograph of the $2 \mathrm{~m}$ telescope of the Thüringer Landessternwarte (TLS). If this stage is successfully passed, the planetary candidate is handed down to SOPHIE at the $1.93 \mathrm{~m}$ telescope at the Observatoire de HauteProvence (OHP). In its high-efficiency mode $(R \approx 40000)$, SOPHIE is able to reach RV precision of a few dozen $\mathrm{m} \mathrm{s}^{-1}$ on a solar-like star, down to $V \approx 14.5 \mathrm{mag}$. This accuracy is fully sufficient for detecting Jupiter-like, and even Saturn-like planets, in a close-in orbit around a solar-like star. We recently also took advantage of the fiber-fed FIES spectrograph attached at the $2.56 \mathrm{~m}$ Nordic Optical Telescope (NOT). The recent refurbishments carried out at this instrument had improved the capability of FIES for very high-precision RV measurements down to $\sim 10 \mathrm{~m} \mathrm{~s}^{-1}$, making this spectrograph a precious resource to use for CoRoT RV follow-up.

The final "step" of the RV follow-up uses high-precision RV measurements with HARPS at ESO's 3.6 m telescope. The $\mathrm{RV}$ follow-up of the faintest candidates in LRa01 $(15 \lesssim V \lesssim$ $16 \mathrm{mag}$ ) has been strengthened using the HIRES echelle spectrograph on the $10 \mathrm{~m}$ Keck I telescope.

The photospheric parameters of the candidates, i.e., effective temperature $\left(T_{\text {eff }}\right)$, gravity $(\log g)$, metallicity $([\mathrm{M} / \mathrm{H}])$, and projected rotational velocity $(v \sin i)$, are usually derived by also analyzing the acquired high-resolution spectra, as already described in other CoRoT exoplanet papers (e.g., Deleuil et al. 2008; Léger et al. 2009; Bruntt et al. 2010; Gandolfi et al. 2010). Stellar masses and radii are then inferred by comparing the location of the objects on a $T_{\text {eff }}$ vs. $\log g$ diagram with theoretical evolutionary tracks. Only an estimate of the spectral type can be derived for candidates with low $S / N$ ratio $(<10-15)$ spectra, as described in some cases in Sect. 6.

For the confirmed planetary candidates high $S / N$ ratio spectra are usually acquired with HARPS, HIRES, and UVES (ESO, Paranal Observatory) spectrographs.

\section{CoRoT planetary candidates}

Here we present the CoRoT transit candidates along with a brief overview over the properties of the star and the detected transit signal. Any follow-up observations that have been performed are also described. The candidates are presented in the following order: confirmed planets, identified non-planetary objects, unsettled good planetary candidates, unsettled low-priority planetary candidates (suspected binaries), false alarms, and the so-called "X-case" candidates (see below).

A planet is considered confirmed when RV-measurements definitely assess the planetary nature of the transiting object and allow to determine its mass (Sect. 6.1). Candidates listed as "settled cases - non-planetary objects" (Sect. 6.2) are objects whose non-planetary nature was identified by either photometric or spectroscopic follow-up observations. These include blends, contaminating eclipsing binaries (CEB), and binary systems.

Most of the observing time reserved for LRa01 was invested in CoRoT-7. Therefore many candidates were being followed-up in the 2009/2010 and some even in the 2010/2011 observing seasons. Still, follow-up observations could not be concluded for all candidates. These are listed as unresolved cases and are subdivided into "unsettled good planetary candidates" (Sect. 6.3) and "unsettled low-priority planetary candidates" (Sect. 6.4), based on the analysis of the CoRoT lightcurves only. The latter were usually not followed-up because of one or more bad characteristics hinting at stellar binary scenario (e.g., out-of-transit variations, depth differences either between even and odd transits or in the three different color channels, very shallow secondary eclipse).

For the sake of completeness, "false alarm" objects are also included (Sect. 6.5). These are shallow transit candidates that were identified by one detection team in lightcurves heavily affected by instrumental effects. They are considered as probable false alarms because they could not be reproduced by other detection teams using different filtering techniques. The "X-case" candidates (Sect. 6.6) are objects that might be planetary candidates if the spectral type of the target star were considerable different than the one listed in Exo-Dat. They have a very low priority in the follow-up program and were not observed so far. Finally, in some cases only a single transit event is present in the CoRoT lightcurve. Those are listed as "mono-transits" (Sect. 6.7). The depth of the signals indicates that these are eclipsing stellar binaries (Table 7).

A concise list of the transit parameters and the follow-up status of the candidates can be found in Tables 5 and 6, respectively. The RV-measurements performed on the CoRoT LRa01 candidates are listed in Table 4. For the RV data of the confirmed exoplanets in LRa01, we refer the reader to the respective articles reporting on their discovery.

\subsection{Confirmed planets}

Four candidates detected during the CoRoT LRa01 run were confirmed as bona fide transiting planets (see Table 2 for the full parameters). Three Jupiter-sized planets, CoRoT-5b (Rauer et al. 2009), CoRoT-12b (Gillon et al. 2010), and CoRoT-21b (Pätzold et al., in prep.), and the first transiting terrestrial planet CoRoT-7b (Léger et al. 2009; Queloz et al. 2009). For the very first time, the bulk density of a small extrasolar planet was derived consistent with the bulk densities of terrestrial planets (see Table 2). An accurate reanalysis of the acquired HARPS and UVES spectra was recently published by Bruntt et al. (2010), leading to an improvement of stellar parameters of CoRoT-7.

There are now a number of independent determinations of the mass of CoRoT-7b. See Table 2 and Hatzes et al. (2011) for a detailed discussion. We note that CoRoT-7b is almost identical to the recently discovered transiting Super-Earth Kepler10b. Kepler-10b has a period of about $P=0.84$ days, mass 
Table 2. Parameters of the planets detected in the CoRoT LRa01 field.

\begin{tabular}{|c|c|c|c|c|c|}
\hline Planet & Host star spectral type & Planetary mass & Planetary radius & Semi-major axis $[\mathrm{AU}]$ & Source \\
\hline \multirow[t]{7}{*}{ CoRoT-7b } & G9V & $4.8 \pm 0.8 M_{\text {Earth }}$ & $1.68 \pm 0.09 R_{\text {Earth }}$ & $0.0172 \pm 0.0002$ & $\begin{array}{l}\text { Léger et al. (2009); } \\
\text { Queloz et al. (2009) }\end{array}$ \\
\hline & G9V & $6.9 \pm 1.5 M_{\text {Earth }}$ & $1.68 \pm 0.09 R_{\text {Earth }}$ & $0.0172 \pm 0.0002$ & $\begin{array}{l}\text { Léger et al. (2009); } \\
\text { Hatzes et al. (2010) }\end{array}$ \\
\hline & G9V & $5.2 \pm 0.8 M_{\text {Earth }}$ & $1.58 \pm 0.10 R_{\text {Earth }}$ & $0.0172 \pm 0.0002$ & $\begin{array}{l}\text { Queloz et al. (2009); } \\
\text { Bruntt et al. (2010) }\end{array}$ \\
\hline & G9V & $2.3 \pm 1.8 M_{\text {Earth }}$ & $1.58 \pm 0.10 R_{\text {Earth }}$ & $0.0172 \pm 0.0002$ & $\begin{array}{l}\text { Pont et al. (2011); } \\
\text { Bruntt et al. (2010) }\end{array}$ \\
\hline & G9V & $8.0 \pm 1.2 M_{\text {Earth }}$ & $1.58 \pm 0.10 R_{\text {Earth }}$ & $0.0172 \pm 0.0002$ & $\begin{array}{l}\text { Ferraz-Mello et al. } \\
\text { (2011); Bruntt et al. } \\
\text { (2010) }\end{array}$ \\
\hline & G9V & $5.7 \pm 2.5 M_{\text {Earth }}$ & $1.58 \pm 0.10 R_{\text {Earth }}$ & $0.0172 \pm 0.0002$ & $\begin{array}{l}\text { Boisse et al. (2011); } \\
\text { Bruntt et al. (2010) }\end{array}$ \\
\hline & G9V & $7.42 \pm 1.21 M_{\text {Earth }}$ & $1.58 \pm 0.10 R_{\text {Earth }}$ & $0.0172 \pm 0.0002$ & $\begin{array}{l}\text { Hatzes et al. (2011); } \\
\text { Bruntt et al. (2010) }\end{array}$ \\
\hline \multirow[t]{4}{*}{ CoRoT-7c* } & G9V & $8.4 \pm 0.9 M_{\text {Earth }} \sin i$ & - & 0.046 & \multirow{4}{*}{$\begin{array}{l}\text { Queloz et al. (2009) } \\
\text { Hatzes et al. (2010) } \\
\text { Ferraz-Mello et al. } \\
\text { (2011) } \\
\text { Boisse et al. (2011) }\end{array}$} \\
\hline & G9V & $12.4 \pm 0.4 M_{\text {Earth }} \sin i$ & - & 0.045 & \\
\hline & G9V & $13.6 \pm 1.4 M_{\text {Earth }} \sin i$ & - & 0.045 & \\
\hline & G9V & $13.2 \pm 4.1 M_{\text {Earth }} \sin i$ & - & 0.045 & \\
\hline CoRoT-7d* & G9V & $16.70 \pm 0.42 M_{\text {Earth }} \sin i$ & - & 0.080 & Hatzes et al. (2010) \\
\hline CoRoT-5b & F9V & $0.467_{-0.024}^{+0.047} M_{\text {Jup }}$ & $1.388_{-0.047}^{+0.046} R_{\text {Jup }}$ & $0.04947_{-0.00029}^{+0.00026}$ & Rauer et al. (2009) \\
\hline CoRoT-12b & G7V & $0.917 \pm 0.07 M_{\text {Jup }}$ & $1.44 \pm 0.13 R_{\text {Jup }}$ & $0.0402 \pm 0.0009$ & Gillon et al. (2010) \\
\hline
\end{tabular}

Notes. ${ }^{(*)}$ CoRoT-7c and CoRoT-7d were detected by RV measurements only. The real nature of CoRoT-7d is not definitely assessed. See Pätzold et al. (in prep.) for the parameters of CoRoT-21b.

$M_{\mathrm{P}}=4.56_{-1.29}^{+1.17} M_{\text {Earth }}$, radius $R_{\mathrm{P}}=1.416_{-0.036}^{+0.033} R_{\text {Earth }}$, and mean density $\rho=8.8_{-2.9}^{+2.1} \mathrm{~g} \mathrm{~cm}^{-3}$ (Batalha et al. 2011).

CoRoT-7b is the only transiting planet in the CoRoT-7b system. Two other planets are inferred from RV measurements only: Queloz et al. (2009) discovered CoRoT-7c; Hatzes et al. (2010) found evidence of the presence of a third planet, CoRoT-7d (see also Table 2), but more RV measurements are required to definitely assess its planetary nature. The detection of these two additional planets is disputed by Pont et al. (2011).

CoRoT-21b, also known as candidate LRa01 E25277 (CoRoT ID 0102725122), has been recently confirmed as a transiting hot-Jupiter planet. The lightcurve of its faint host star $(V=16.1 \mathrm{mag})$ contains a $0.45 \%$ deep transit signal with a period of 2.73 days (Fig. 6). Photometric follow-up observations performed with IAC 80 confirm the transit event on target. Radial velocity measurements acquired with HARPS and HIRES show significant variations in phase with the CoRoT ephemeris that are consistent with a $\sim 2 M_{\text {Jup }}$ planet around an F8 IV $\operatorname{star}\left(T_{\mathrm{eff}} \approx 6100 \mathrm{~K}, \log g \approx 3.5 \mathrm{dex}\right)$. Since the parameters of CoRoT-21b are still under investigation, the planet is not listed in Table 2 but in Tables 5 and 6 . It will be presented in a forthcoming paper (Pätzold et al., in prep.), along with the HARPS and HIRES RV measurements.

\subsection{Settled cases: non-planetary objects}

The following objects are identified as non-planetary objects based on ground-based follow-up observations.

\subsubsection{LRa01 E1 $0544-\mathrm{CHR}-0102714746$}

LRa01 E1 0544 is a relatively bright $\operatorname{star}(V=13.39 \mathrm{mag})$ with a $0.15 \%$ deep eclipse occurring every 2.75 days. AAOmega observations classify the target as an F7 dwarf star, in good agreement with the classification listed in Exo-Dat (F8 IV). SOPHIE spectroscopic observations indicate a fast rotator with no significant RV variations down to a precision of $50 \mathrm{~m} \mathrm{~s}^{-1}$. EulerCam measurements show no transit events on target. Instead a $\sim 4$ mag fainter star located $9^{\prime \prime}$ west of the main target has deep eclipses ( $D \approx 20 \%$ ). IAC 80 observations confirm this result. This object is a contaminating eclipsing binary (CEB).

\subsubsection{LRa01 E1 $0561-\mathrm{CHR}-0102597681$}

This is a relatively bright candidate $(V=12.00 \mathrm{mag})$ listed as an A0 V star in Exo-Dat. The CoRoT lightcurve shows a $0.70 \%$ deep transit occurring every 20.82 days superimposed on a $\gamma$-Doradus like pulsations. Low-resolution AAOmega 

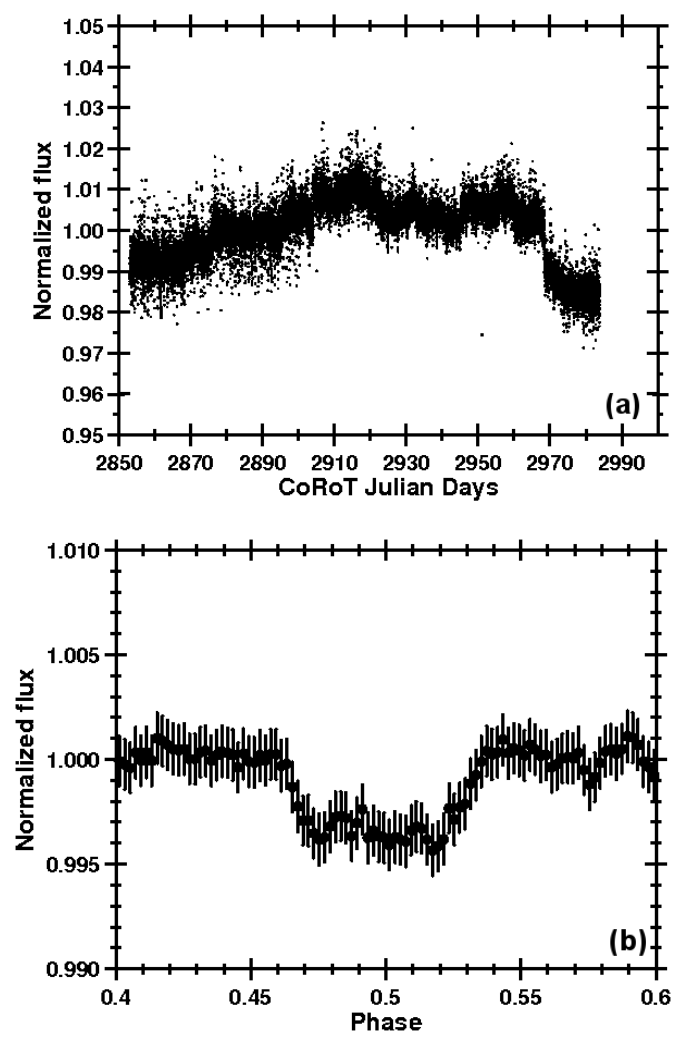

Fig. 6. Top panel: raw white lightcurve of LRa01 E2 5277 (also known as CoRoT-21b). Bottom panel: normalized and phase-folded raw white lightcurve of the transit candidate LRa01 E2 5277 at the transit period $P=2.73$ days. A baseline fit was subtracted around each transit before folding.

spectroscopy classifies this object as an A7IV/V star. Two moderate $S / N \approx 35$ ratio SOPHIE spectra reveal a lowcontrast single peak cross-correlation function (CCF) with an $\mathrm{RV}$ variation of about $52 \mathrm{~km} \mathrm{~s}^{-1}$, in anti-phase with the CoRoT ephemeris, i.e., the eclipses occur on the rising part of the RV curve and are caused by the star whose CCF peak is detected in the SOPHIE spectra. A single epoch UVES spectrum with higher $S / N$ ratio $(\sim 120)$ unveils the presence of other two components in the system, making the candidates a spectroscopically resolved SB3 system with pulsating components.

\subsubsection{LRa01 E1 2890 - MON - 0102618931}

According to Exo-Dat, this candidate has an apparent $V$-magnitude of 15.73 and its spectral type is G5 III. IAC 80 onoff photometry shows that a contaminating binary with a $40 \%$ deep eclipse is the origin of the transit signal in the CoRoT lightcurve $(D=0.29 \%, P=2.43$ days $)$. It is $\sim 3.2 \mathrm{mag}$ fainter than the target and positioned $\sim 12^{\prime \prime}$ southeast from LRa01 E1 2890.

\subsubsection{LRa01 E1 3666 - MON - 0102790970}

This is a faint ( $V=15.47 \mathrm{mag}$ ) F5 V star (Exo-Dat) with a $0.45 \%$ deep transit signal occurring every 1.55 days. CFHT and IAC 80 observations identify a nearby background eclipsing binary located $\sim 8^{\prime \prime}$ west of the target as the source of the signal. It is $\sim 0.5$ mag fainter than LRa01 E1 3666 and exhibits a $1.5 \%$ deep eclipse. Taking into account the light contamination inside the photometric mask, the observed variation is consistent with

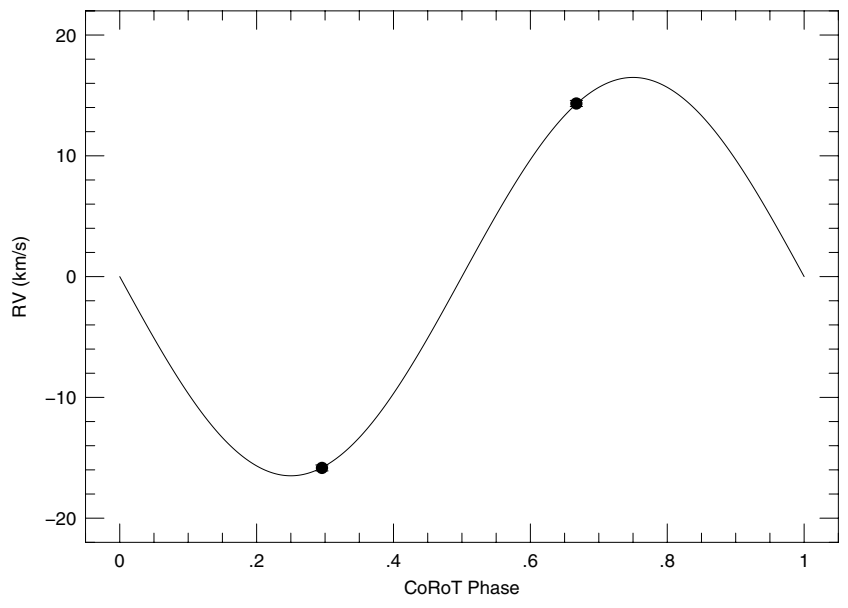

Fig. 7. HARPS RV measurements of LRa01 E1 5015 (points) phased to the CoRoT transit period and epoch. The best-fitting sine curve is overplotted with a line. Note that the systemic radial velocity $V_{\gamma}=$ $78.517 \mathrm{~km} \mathrm{~s}^{-1}$ has been subtracted.

the CoRoT transit-like signal. The candidate is a contaminating eclipsing binary (CEB).

\subsubsection{LRa01 E1 5015 - MON - 0102777869}

This is a candidate around a relatively faint $(V=16.17 \mathrm{mag})$ G2 V star (Exo-Dat) with a $1 \%$ deep transit signal and a period of 13.69 days. The long transit duration of about $10 \mathrm{~h}$ suggests an eclipsing binary scenario.

ESA-OGS observations show that the transit is on target. Figure 7 reports the HARPS RV measurements of LRa01 E1 5015 phase folded to the CoRoT transit period and epoch, along with a sine fit. For a circular orbit the semiamplitude is $K=16.3 \mathrm{~km} \mathrm{~s}^{-1}$, which corresponds to a mass function $^{4} f(m)=0.00614 M_{\odot}$. Assuming a mass of $1 M_{\odot}$ for the main component, the companion mass is $\approx 0.18 M_{\odot}$. This is an SB1 system with a low-mass companion star.

\subsubsection{LRa01 E1 $4353-\mathrm{MON}-0102692038$}

This candidate in the lightcurve of a $V=15.78$ mag star of spectral type A5 V (Exo-Dat) has a 1.09\% deep transit signal with a period of 5.23 days. EulerCam on-off photometry shows a constant flux in the target but a 5\% deep eclipse in a contaminant star (CoRoT ID: 0102691690, $V=16.7 \mathrm{mag}$ ). IAC 80 confirms the EulerCam observations. This is a contaminating eclipsing binary (CEB).

\subsubsection{LRa01 E2 $1123-\mathrm{MON}-0102615551$}

A $1.80 \%$ deep signal with a period of 3.88 days is detected in the CoRoT lightcurve of this candidate. IAC 80 and Wise photometry confirms the transit signal is on target. UVES and HARPS observations of this $V=14.62$ mag object identify the target as a K5 dwarf star. Two CORALIE and six

\footnotetext{
${ }^{4}$ We remind the reader that the mass function expressed in solar units is defined as $f(m)=M_{2}^{3}\left(\sin ^{3} i\right) /\left(M_{1}+M_{2}\right)^{2}=\left(1.03608 \times 10^{-7}\right)(1-$ $\left.e^{2}\right)^{3 / 2} K_{1}^{3} P$, where $M_{1}$ and $M_{2}$ are the masses in solar units of the primary and secondary component, $i$ and $e$ the orbit inclination and eccentricity, $K_{1}$ the RV semi-amplitude of the primary star in $\mathrm{km} \mathrm{s}^{-1}$, and $P$ the orbital period expressed in days.
} 
HARPS RV measurements show no significant sinusoidal variations with an amplitude greater than $\sim 50 \mathrm{~m} \mathrm{~s}^{-1}$.

An examination of the $\mathrm{Ca}$ II $\mathrm{H} \& \mathrm{~K}$ region from spectra taken with UVES shows three emission components. The RVs of two components vary in phase with twice the transit period and with a maximum velocity difference of about $67 \mathrm{~km} \mathrm{~s}^{-1}$. This is a blend scenario: a probable hierarchical triple system consisting of an active $\mathrm{K} 5 \mathrm{~V}$ primary orbited by two eclipsing active M-type stars that are too faint to be seen in the metallic lines used for the RV measurements.

\subsubsection{LRa01 E2 1145 - CHR - 0102707895}

A transit with the following parameters is detected in the CoRoT lightcurve: $D=0.43 \%$ and $P=5.78$ days. The $V=13.96$ mag target is already known from the CoRoT IRa01 run (IRa01 E1 1873; Carpano et al. 2009) and is classified as an A9IV/V star on the basis of AAOmega observations. SOPHIE measurements spectroscopically resolve the target as an SB1 system with an RV curve in anti-phase with the CoRoT ephemeris. Assuming a circular orbit, the RV curve semi-amplitude of the eclipsing star is $K=23.5 \mathrm{~km} \mathrm{~s}^{-1}$.

\subsubsection{LRa01 E2 1897 - MON - 0102658070}

This $V=14.72$ mag candidate, classified as an F2 II star according to Exo-Dat, shows a deep transit-like signal $(D=2.80 \%)$ with a period of 4.67 days. Hints of a secondary eclipse are detected in the CoRoT lightcurve at phase $=0.5$. CFHT photometric observations show that the signal originates from an eclipsing binary located $\sim 3^{\prime \prime}$ northeast from the CoRoT main target. The contaminant exhibits flux variation of about $7.8 \%$ occurring at the predicted CoRoT ephemeris. This is consistent with the transit signal when the dilutions by the main target and by a second brighter star located 24.4" southwest are considered. This candidate is a contaminating eclipsing binary (CEB).

\subsubsection{LRa01 E2 2249 - CHR - 0102755837}

This candidate has a $0.38 \%$ deep transit occurring every 27.93 days. Low-resolution spectra obtained with AA0mega classify the star ( $V=13.88 \mathrm{mag})$ as $\mathrm{K} 0 \mathrm{III} / \mathrm{IV}$, in good agreement with the Exo-Dat spectral type (K0 III). SOPHIE RV measurements show the candidate to be an SB1 system $\left(K=12.2 \mathrm{~km} \mathrm{~s}^{-1}\right.$ if $e=0$ ). Figure 8 reports the RV measurements with a sine fit using the CoRoT transit period and epoch. The mass function for the system is $f(m)=0.00526 M_{\odot}$. Assuming a primary mass of $1 M_{\odot}$, this results in a secondary mass of $0.17 M_{\odot}$.

\subsubsection{LRa01 E2 $2481-\mathrm{CHR}-0102723949$}

The CoRoT lightcurve of this candidate $(V=13.96$ mag, $\mathrm{SpT}=$ F5 III; Exo-Dat) contains transit-like signals with a depth of $1.20 \%$ and a period of 51.76 days. It is also found in the IRa01 run (IRa01 E1 2046) and listed as a mono-transit candidate in the IRa01 report papers (Carpano et al. 2009; Moutou et al. 2009). The spectral classification based on low-resolution spectroscopy performed with AAOmega hints at a stellar binary. According to the method described in Gandolfi et al. (2008), the spectral type of this object "changes" from G0V to G8 V as a function of the fitted spectral region, suggesting the presence of two stellar objects whose lines are blended in the low-resolution AAOmega spectrum. A single epoch SOPHIE spectrum shows

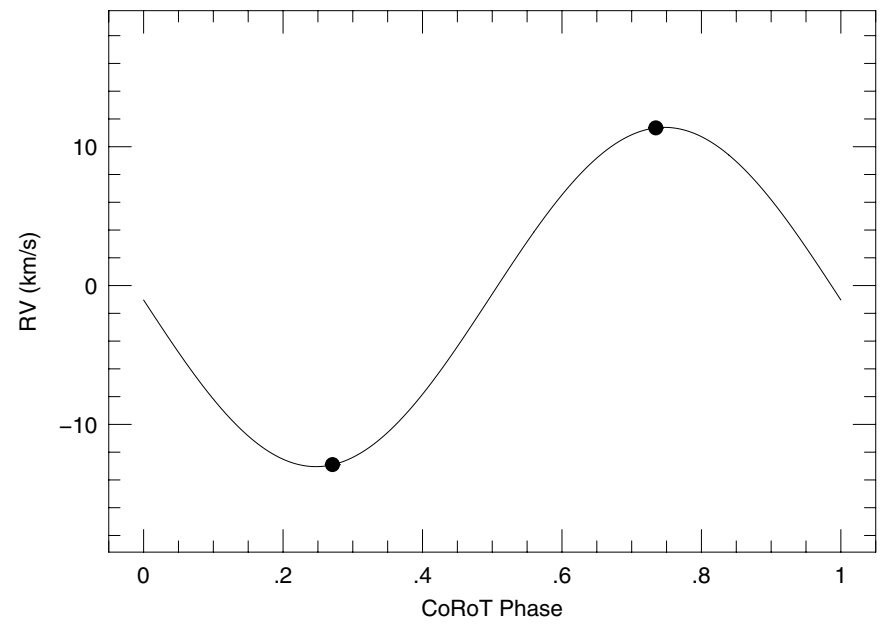

Fig. 8. SOPHIE RV measurements of LRa01 E2 2249 (points) phased to the CoRoT transit period and epoch. The best-fitting sine curve is overplotted with a line. Note that the systemic radial velocity $V_{\gamma}=$ $48.401 \mathrm{~km} \mathrm{~s}^{-1}$ has been subtracted.

an SB2 system (Moutou et al. 2009), confirming the AAOmega binary scenario.

\subsubsection{LRa01 E2 2694 - CHR - 0102590741}

Transit-like events with $1.30 \%$ depth and a 30.40 days period are found in the lightcurve of this $\operatorname{star}(V=13.56 \mathrm{mag}, \mathrm{SpT}=\mathrm{A} 0 \mathrm{IV}$; Exo-Dat). SOPHIE and HARPS spectra show no CCF and only He $\mathrm{I}$ absorption and strong emission Balmer lines, indicative of a Be-type star. Low-resolution AAOmega spectra confirm the SOPHIE results and yield a spectral type of B3Ve, which translates into a stellar radius of about $5 R_{\odot}$ (Cox 2000). Taking into account the contamination level for this star ( $\sim 14 \%$ according to Exo-Dat), the observed transit, if on target, is therefore caused by a stellar object with a radius of about $0.6 R_{\odot}$.

\subsubsection{LRa01 E2 4129 - MON - 0102590008}

The $V=15.71 \mathrm{mag}$ G0 IV target star (Exo-Dat) has two faint nearby contaminants located about $4.5^{\prime \prime}$ to the north-northeast. Wise observations are inconclusive. EulerCam photometric observations show no signs of transits on the main target. Instead a $7 \%$ drop in brightness is found in one of the two contaminant stars, which accounts for the transit events observed on LRa01E24129 (0.18\% deep eclipses every 1.94 days). Therefore, this case is classified as a contaminating eclipsing binary (CEB).

\subsubsection{LRa01 E2 5084 - MON - 0102667981}

This $V=15.95$ mag transit candidate is classified as an A5 subgiant star according to Exo-Dat. The transit is $0.28 \%$ deep with a 9.92 days period. Based on HARPS observations, the candidate is an SB1 system. Assuming a circular orbit, the HARPS measurements yield a $K=37.2 \mathrm{~km} \mathrm{~s}^{-1} \mathrm{RV}$ curve in anti-phase with the CoRoT ephemeris.

\subsubsection{LRa01 E2 5184 - CHR - 0102779966}

This $V=15.41 \mathrm{mag}$ candidate $(D=0.41 \%, P=7.37$ days $)$, classified as $\mathrm{K} 2 \mathrm{~V}$ star in Exo-Dat, is already known from the 


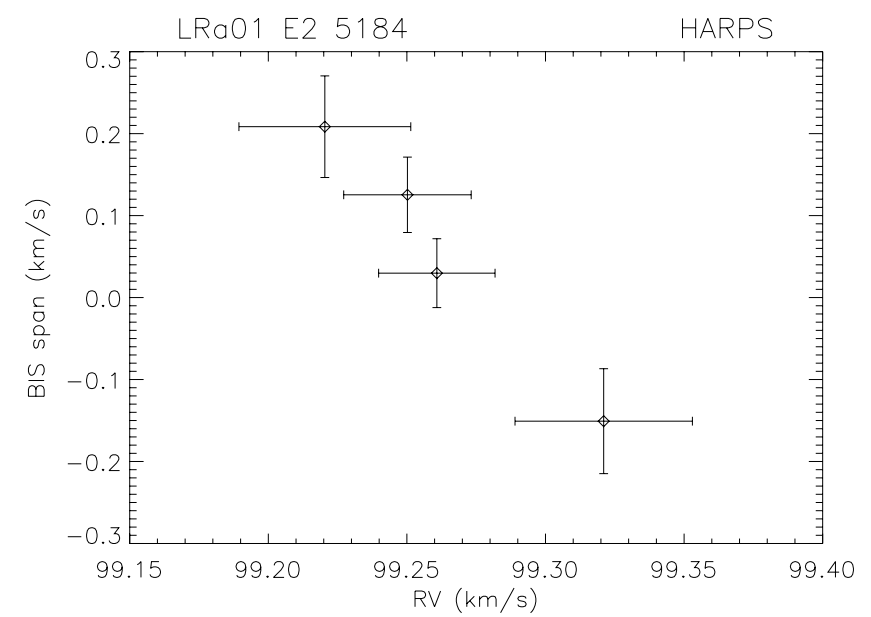

Fig. 9. CCF bisector spans versus RV measurements as derived from the HARPS spectra of LRa01 E2 5184.

IRa01 field as IRa01 E14108 Carpano et al. (2009); Moutou et al. (2009). CFHT ground-based photometry confirms the transit to be on target. HARPS spectra yield $T_{\mathrm{eff}}=5000 \pm 100 \mathrm{~K}$, $\log g=4.4 \pm 0.1 \mathrm{dex},[\mathrm{M} / \mathrm{H}]=0.07 \pm 0.06 \mathrm{dex}, v \sin i=1.5 \pm$ $0.5 \mathrm{~km} \mathrm{~s}^{-1}$, and $\mathrm{SpT}=\mathrm{K} 0 \mathrm{~V}$. HARPS data also indicate a strong bisector-RV correlation consistent with a blended eclipsing binary (i.e., diluted triple system or background/foreground eclipsing binary), as shown in Fig. 9.

\subsubsection{LRa01 E2 5747 - MON - 0102753331}

This candidate $(V=16.16 \mathrm{mag})$ is already known from the IRa01 field as IRa01 E1 4617 (Carpano et al. 2009; Moutou et al. 2009). The transit is $3.64 \%$ deep, appears every 19.75 days, and has a duration of $14.13 \mathrm{~h}$ suggesting a stellar companion. No CCF is detected with HARPS. The spectrum shows only broad Balmer and $\mathrm{Mg} \mathrm{I}-\mathrm{b}$ lines, indicative of a rapidly rotating A-type star, in agreement with the Exo-Dat spectral classification (A5 IV). Even assuming a late A-type dwarf star, the stellar radius would be too large $\left(R_{*}>1.5 R_{\odot} ;\right.$ Cox 2000) to make the observed $3.64 \%$ deep transit of planetary origin. Therefore the transiting object, if on target, is a stellar companion.

\subsubsection{LRa01 E2 $3739-$ MON - 0102755764}

The CoRoT lightcurve of this candidate ( $V=15.55 \mathrm{mag})$ shows a V-shaped, $2.93 \%$ deep transit signal with a duration of $6.97 \mathrm{~h}$ and a period of 61.48 days, superimposed on a low-amplitude pulsation (Debosscher et al. 2009). Shape, duration, and depth suggest grazing eclipses of an evolved star by a stellar companion. A single transit event was already observed in the IRa01 field (IRa01E14014), whereas two transits are found in the LRa01 lightcurve. EulerCam observations confirm the transit to be on target but 0.14 days after the predicted ephemeris. Taking into account the transit timing error of about 0.07 days at the time of the EulerCam follow-up (25 January 2009), the observed transit occurred only $2 \sigma$ after the predicted event. Nearby stars were either stable during the observation or their variation was too small to account for the transit signal detected in the CoRoT lightcurve. Therefore the transit is concluded to be on target. HARPS observations show no CCF for this target. Only broad Balmer lines are visible in the HARPS spectrum. This is consistent with the star being a rapidly rotating A-type star, in agreement with the Exo-Dat A5 IV spectral type. As described in Sect. 6.2.16, an eclipsing stellar companion star is suspected to orbit around this candidate and follow-up observations are completed.

\subsubsection{LRa01 E2 5756 - MON - 0102582529}

A $2.72 \%$ deep transit with a period of 15.84 days is found in the CoRoT lightcurve of this $V=16.24$ mag candidate, whose spectral type is F0 V according to Exo-Dat. The transit signal is rather deep and the ingress/egress steeper than expected for a planetary candidate. But it is not ruled out as a binary on the photometric level, because the transit can also be produced by a planet with a higher impact parameter.

IAC 80 photometry establishes that the transit event is on target. Spectral observations with HARPS reveals no CCF. The spectrum is consistent with LRa01 E2 5756 being a rapidly rotating A-type star. No additional follow-up observations are planned because the transiting object is suspected to be a stellar companion.

\subsection{Unsettled good planetary candidates}

\subsubsection{LRa01 E1 0286 - CHR - 0102742060}

A shallow eclipse $(0.03 \%)$ in the lightcurve of this relatively bright star $V=13.30 \mathrm{mag}$ was discovered at a period of 3.60 days (Fig. 10).

Initially, this candidate was regarded as a possible 1.9 $R_{\text {Earth-sized planet around a main-sequence solar-like }}$ star. However, reconnaissance spectroscopy performed with Sandiford in February 2009 suggests that the target is a G8/9 IV star. Additional observations with HIRES and HARPS indicate $T_{\text {eff }}=5250 \pm 80 \mathrm{~K}, \log g=3.75 \pm 0.10 \mathrm{dex},[\mathrm{M} / \mathrm{H}]=-0.10 \pm$ $0.05 \mathrm{dex}$, and $v \sin i=3.0 \pm 1.0 \mathrm{~km} \mathrm{~s}^{-1}$, compatible with an evolved star of $M_{*} \approx 1.1 M_{\odot}$ and $R_{*} \approx 2.3 R_{\odot}$. This excluded a terrestrial-sized object but a transiting planet with $R_{\mathrm{P}} \approx 4.4 R_{\text {Earth }}$ was still possible.

Radial velocity observations carried-out over two years with SOPHIE, HIRES, HARPS, FIES, and Sandiford revealed a longterm RV trend indicative of an SB1 not in phase with the CoRoT transit ephemeris. The orbital fit to the data is shown in Fig. 11. The orbit seems nearly circular $(e \approx 0.01)$ with a period of $P=$ $337.52 \pm 0.20$ days and has an RV semi-amplitude $K=6.22 \pm$ $0.18 \mathrm{~km} \mathrm{~s}^{-1}$. The derived mass function $f(m) \approx 0.0084 M_{\odot}$ implies a companion mass of approximately $0.22 M_{\odot}$ assuming $1.1 M_{\odot}$ for the primary star's mass.

The RV residual to the binary orbit fit have an rms scatter of about $7.5 \mathrm{~m} \mathrm{~s}^{-1}$ when using only the higher quality HARPS and HIRES data. Figure 12 shows the RV measurement residuals phased to the CoRoT transit period. There is no convincing sinusoidal variations. The RV semi-amplitude of a possible planetary companion orbiting the target star would have to be less than about $5 \mathrm{~m} \mathrm{~s}^{-1}$.

Recent time-series photometric observations performed with MegaCam installed on CFHT3.6 m and EulerCam on Euler1.2 m suggest that a faint, nearby $\operatorname{star}\left(R \approx 18 \mathrm{mag}, 5^{\prime \prime}\right.$ south from the target) might experience eclipses of $\sim 5 \%$ that would account for the detected $0.03 \%$ transit-like signal on LRa01 E1 0286. However, the photometric follow-up is still not conclusive and additional investigations are needed to assess the real nature of the candidate. In conclusion, the target star is part of a longperiod binary and the transit signal remains unresolved. The 

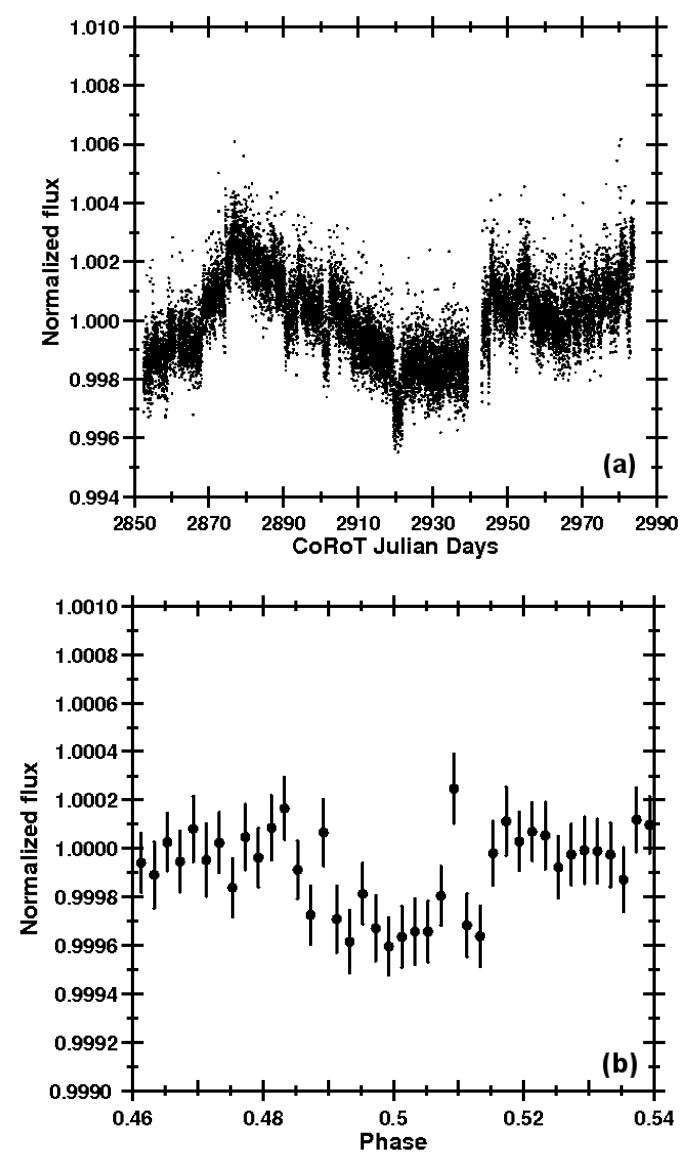

Fig. 10. Top panel: raw white lightcurve of LRa01 E1 0286, which shows instrumental effects (jumps). Bottom panel: normalized and phase-folded white lightcurve of the transit candidate LRa01 E1 0286 at the period $P=3.60$ days after filtering with ExoTrans (Grziwa et al. in prep.). Additional filtering was necessary to make the transit visible in this example.
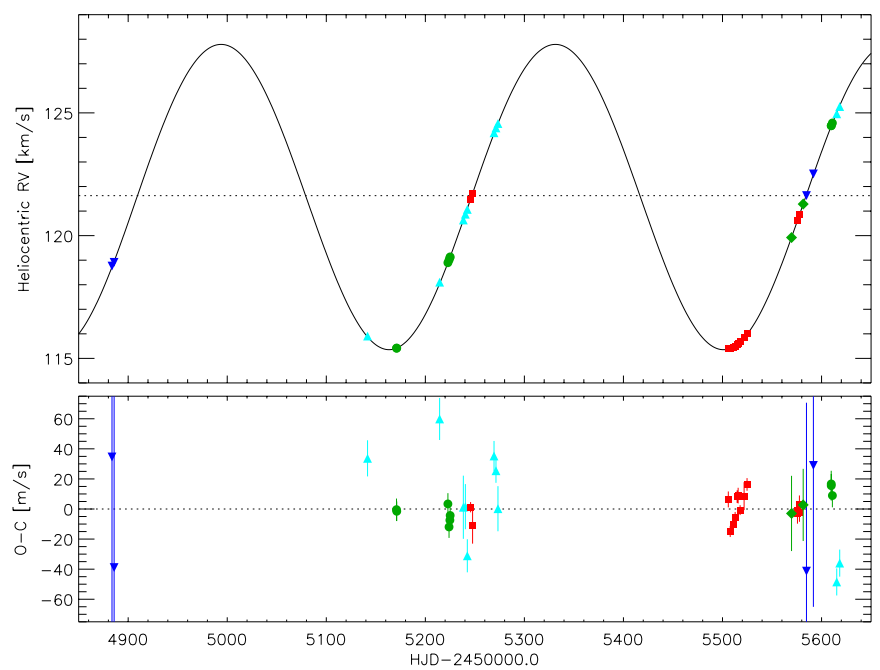

Fig. 11. Top panel: radial velocity curve of LRa01 E1 0286 as observed with Sandiford (blue downward triangles), SOPHIE (light blue upward triangles), HIRES (green circles), HARPS (red squares), and FIES (green diamonds). The solid line represents the best-fit Keplerian orbit to the data (see text for more details). An RV shift was let free to vary in the fit between the five data-sets. The systemic RV of $V_{\gamma}=$ $121.603 \mathrm{~km} \mathrm{~s}^{-1}$, as derived from the HARPS data-set only, is plotted with a horizontal dotted line. Bottom panel: the RV residuals after subtracting the orbital solution (see the online edition of the journal for a color version of this figure).

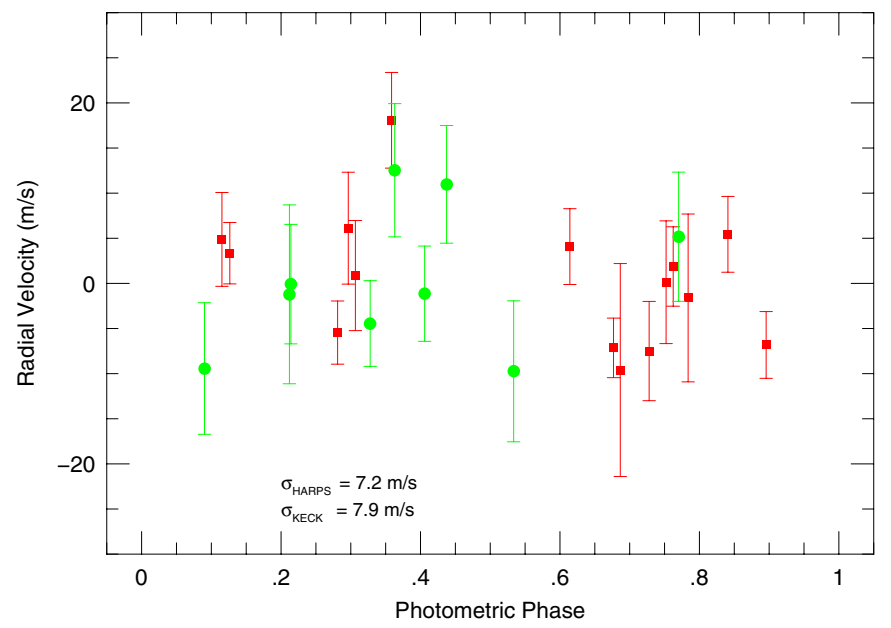

Fig. 12. HARPS (red squares) and HIRES (green circles) RV residuals of LRa01 E1 0286 phased to the CoRoT transit period $P=3.60$ days and epoch. The root main squares to the binary orbit fit, when considering only the HARPS and HIRES data, are reported in the labels (see the online edition of the journal for a color version of this figure).

LRa01 E1 0286 candidate might be either a planet in a stellar binary system or a contaminating eclipsing binary.

\subsubsection{LRa01 E1 $2101-\mathrm{CHR}-0102568803$}

According to Exo-Dat, this $V=14.15 \mathrm{mag}$ target is of spectral type K1 III. The CoRoT lightcurve shows a $2 \%$ sun-spot-induced variability with a period of $\sim 11$ days. LRa01 E1 2101 appears to be orbited by a transiting object ( $D=0.08 \%, P=2.72$ days). The signal is only significant in the red CoRoT photometric channel. As already described in Sect. 3, owing to the star's PSF on the CCD and the choice of the photometric mask, the expected depth of the transit in the green and blue CoRoT photometric channels is lower than the scatter of the data-points, preventing any comparison of the transit depth in the different channels. Therefore, it cannot be concluded from CoRoT data alone that the candidate is a contaminating eclipsing binary. The first HARPS spectrum reveals a narrow single peak CCF $\left(F W H M=8 \mathrm{~km} \mathrm{~s}^{-1}\right)$ and a K6V star with $T_{\text {eff }} \approx 4250 \mathrm{~K}$ and $\log g \approx 4.5 \operatorname{dex}\left(M_{*} \approx 0.7 M_{\odot}, R_{*} \approx 0.7 R_{\odot}\right)$. The shallow transit depth thus implies a companion radius of about $2 R_{\oplus}$. But the transit is V-shaped, suggesting a grazing transit/eclipse. Combined measurements with CFHT and MONET-North exclude background eclipsing binaries. The transit is considered to be on target. Six RV measurements acquired with HARPS show no significant sinusoidal variation down to a precision of $18 \mathrm{~m} \mathrm{~s}^{-1}$. Follow-up is ongoing.

\subsubsection{LRa01 E1 $2240-\mathrm{CHR}-0102698887$}

The transit signal occurs every 2.03 days in the lightcurve of this $V=15.22$ mag target, classified as an F8 subgiant according to Exo-Dat. The shallow (0.09\% deep) signal is a little asymmetric when phase-folded. However, owing to the low $S / N$ ratio, the transit shape cannot be used to rule out a no-planet scenario. If the candidate is a planet, the transit depth suggests a Neptune-like planetary radius. No follow-up observations have been made yet. 


\subsubsection{LRa01 E1 $3216-\mathrm{MON}-0102754163$}

This is a faint $V=15.7$ mag A5 IV candidate (Exo-Dat) with a periodic 3.11 days transit signal of depth $0.13 \%$. It shows shallow out-of-transit variations. No ground-based follow-ups have been performed yet.

\subsubsection{LRa01 E1 3221 - MON - 0102634864}

This is a candidate with a fairly long period $P=32.33$ days. The transit is $\mathrm{V}$-shaped and $2.33 \%$ deep. This target of brightness $V=15.58 \mathrm{mag}$ is listed in Exo-Dat as an A5 dwarf star. However, pulsations with periods of 0.78 and 8.75 days typical for giant stars have been detected in the CoRoT lightcurve, in disagreement with the main-sequence scenario. No follow-up observations have been made.

\subsubsection{LRa01 E1 4423 - MON - 0102782651}

A faint $(V=16.22 \mathrm{mag})$ candidate showing a V-shaped shallow transit $(D=0.25 \%)$ with a period of 1.87 days. No followup observations have been carried out. The CoRoT lightcurve is strongly affected by instrumental effects (jumps). Exo-Dat list the spectral type as $\mathrm{K} 4 \mathrm{~V}$.

\subsubsection{LRa01 E1 4594-MON - 0102617334}

This transiting candidate $(D=0.27 \%)$ was not spectroscopically observed owing to the faintness of the target star $(V=$ $16.66 \mathrm{mag}, \mathrm{SpT}=\mathrm{O} 8 \mathrm{III}$ from Exo-Dat). IAC 80 photometry excludes background eclipsing binaries. The transit duration of $6.62 \mathrm{~h}$ for a transit period of $P=5.49$ days is consistent with an evolved host star.

\subsubsection{LRa01 E1 4667 - MON - 0102588881}

A $1.52 \%$ deep transit signal with a period of 27.29 days is found in the lightcurve of this $V=16.08$ mag star of spectral type A5 IV (Exo-Dat). Wise photometric observations are inconclusive because of bad weather. IAC 80 observations exclude contaminating eclipsing binaries. Two RV measurements acquired with HARPS at photometric phases 0.43 and 0.76 shows a radial velocity variation of $84 \mathrm{~m} \mathrm{~s}^{-1}$, which is comparable to the errors (i.e., $\sim 70 \mathrm{~m} \mathrm{~s}^{-1}$ ). The HARPS spectra unveil a G0 V star, in disagreement with Exo-Dat. Assuming $M_{*}=1 M_{\odot}$ for the host-star, a Jupiter-mass planet in a 27.29 days circular orbit around LRa01 E1 4667 would produce a peak-to-peak $\mathrm{RV}$ variation of $\approx 120 \mathrm{~m} \mathrm{~s}^{-1}$, which is almost twice the HARPS errors. Although the RVs exclude a stellar/brown dwarf companion to LRa01 E1 4667, considerably more HARPS measurements are required to assess the real nature of the transiting object. Follow-up is ongoing.

\subsubsection{LRa01 E1 4719 - MON - 0102703155}

A 1.26 days period transit signal of depth $0.10 \%$ is found in the CoRoT light curve of this $V=15.88 \mathrm{mag}$ candidate $(\mathrm{SpT}=$ F8 IV, Exo-Dat). The transit is V-shaped and asymmetric when phase-folded. However, the $S / N$ ratio of the detected events is low. The shape may be distorted by either stellar activity or photometric noise. EulerCam could not observe the transit on target. This is expected given the shallow transit depth. Although the transit timing error at the time of the EulerCam observations
(28 October 2010) was $2 \mathrm{~h}$ and there is a risk that the transit might have been missed, it is concluded from photometric on-off observations that large variations by contaminants are probably not the cause for the transit event. No RV-measurements have been acquired.

\subsubsection{LRa01 E1 4820 - MON - 0102751316}

This is a transiting candidate with a depth of $0.46 \%$ and a period of 1.61 days. Photometric follow-up with the ESA-OGS facility confirms that the transit is on target. Analysis of the lightcurve indicates possible depth differences between even and odd transits and out-of-transit variation. The A5 IV star (Exo-Dat) is too faint $(V=16.15 \mathrm{mag})$ for RV confirmation.

\subsubsection{LRa01 E1 5320 - MON - 0102666452}

This is another transiting candidate around a faint star $(V=$ $16.13 \mathrm{mag}$, SpT = G2 V; Exo-Dat). The $0.14 \%$ deep signal can only be identified when phase-folded with a 1.97 days period. The $3.3 \mathrm{~h}$ transit duration appears to be a little long for a planetary object. No ground-based observations have been performed.

\subsubsection{LRa01 E1 5536 - MON - 0102670085}

The CoRoT lightcurve of this $V=16.21$ mag star of spectral type F8 IV (Exo-Dat), shows a $0.27 \%$ deep transit signal occurring every 0.90 days. CFHT photometric observations find a $0.40 \pm 0.25 \%$ deep transit on target, compatible with the CoRoT signal. However, some background stars, spatially located around the target, cannot be excluded as possible contaminants and the observations are considered inconclusive. No spectroscopic data have been acquired.

\subsubsection{LRa01 E2 $3156-\mathrm{CHR}-0102716818$}

The target ( $V=15.76 \mathrm{mag}$ ) is a K2 III star according to Exo-Dat. The $0.15 \%$ deep transit signal appears every 1.47 days and is detected only in the red CoRoT channel. As for LRa01 E1 2101 (Sect. 6.3.2), also in this case the photo-noise in the green and blue channels is higher than the expected depth. The transit is $\mathrm{V}$-shaped, and the duration of $\sim 2 \mathrm{~h}$ is quite long for a planet. Observations with IAC 80 exclude contamination by neighboring objects. The transit is likely on target with a $20-30 \%$ chance of a missed transit owing to timing error. Two and seven RV measurements with HARPS and HIRES, respectively, show no significant variations down to a precision of $10 \mathrm{~m} \mathrm{~s}^{-1}$. The candidate is still under investigation.

\subsubsection{LRa01 E2 3619 - MON - 0102765275}

V-shaped transit signals of $6 \%$ depth with a period of 50.91 days are found in the lightcurve of this star $(V=15.56 \mathrm{mag})$. The spectral type is $\mathrm{G} 8 \mathrm{~V}$, according to Exo-Dat. Low-resolution spectroscopy performed with AAOmega indicates that the target is a G0IV/V star. However the CoRoT photometric data show multi-periodic variations that seem more consistent with a giant. Therefore, the true spectral type is unclear. This candidate is also found in the IRa01 field (as IRa01 E1 2060; Carpano et al. 2009). No additional ground-based follow-ups have been performed. 


\subsubsection{LRa01 E2 4519 - MON - 0102580137}

A $0.14 \%$ deep candidate with a period of 2.37 days is detected in the lightcurve of this A5 IV star (Exo-Dat) with magnitude $V=15.75 \mathrm{mag}$. EulerCam and IAC 80 find no relevant photometric variations in any of the nearby stars. However, the transit may have been missed owing to the timing errors $(1.5 \mathrm{~h})$ at the time of the EulerCam and IAC 80 observations (November-December 2010). Still, the transit is considered to be likely on target. No RV measurements have been acquired for this star.

\subsection{Unsettled low-priority planetary candidates}

Many likely binary candidates are discovered at the photometric level and have a low priority in the follow-up observation chain. Consequently, many of these were not observed by the follow-up team, especially when the target star is faint.

\subsubsection{LRa01 E1 2970 - CHR - 0102625386}

The $V=14.49$ mag candidate shows a V-shaped, $0.62 \%$ deep transit signal occurring every 34.10 days. The transit is only seen in the CoRoT red channel. No event is detected in the blue and green channels at 4 and $5 \sigma$ significance, respectively. This is a characteristic sign of a contaminant eclipsing binary. Hints of secondary eclipse are also detected in the red lightcurve. ExoDat lists the spectral type as A5 IV. No follow-up observations have been performed for this star because the candidate is suspected to be a contaminating eclipsing binary (CEB).

\subsubsection{LRa01 E1 $3617-\mathrm{MON}-0102617210$}

According to Exo-Dat this is an A0 V star $(V=15.62 \mathrm{mag})$. The $0.64 \%$ deep signal has a period of 2.73 days. Several coherent frequencies are found, which hint at stellar variability induced by a massive companion. Hints of secondary shallow eclipses have been recently found in the CoRoT lightcurve. No follow-up observations were carried out because a binary system is suspected for this candidate.

\subsubsection{LRa01 E1 $3674-\mathrm{CHR}-0102732757$}

A V-shaped transit signal is found in the lightcurve of this A0 V star $(V=15.32 \mathrm{mag}$, Exo-Dat). The transit duration of $3.38 \mathrm{~h}$ is quite long for a 1.97 days transit period. The $0.20 \%$ deep signal can only be seen in the red CoRoT channel. It is not detected in both green and blue channel, with a $\sim 3 \sigma$ significance. No follow-up observations have been performed on this candidate because a CEB scenario is suspected.

\subsubsection{LRa01 E1 $4272-$ MON - 0102626872}

The detected transit of this object is $2.44 \%$ deep with a 1.88 days period. In Exo-Dat the star is listed as A0 V with a brightness of $V=15.87$ mag. Photometry with ESA-OGS shows the transit to be on target. Owing to out-of-transit variations in the lightcurve a stellar binary is suspected.

\subsubsection{LRa01 E1 4777 - CHR - 0102620061}

A V-shaped transit signal with a period of 3.35 days and a depth of $1.96 \%$ is detected in the lightcurve of this $V=15.26 \mathrm{mag}$ candidate (A5 IV, Exo-Dat). The duration (3.90 h) is quite long for a V-shaped transit and the depths observed in the three CoRoT colors differ by more than $1 \sigma$. IAC 80 observations performed in February 2011 show no variations on target or on any of the nearby stars. An underestimate of the transit timing error, listed at about $30 \mathrm{~min}$ at the time of the IAC 80 observations, might account for the ground-based transit non-detection. No RV follow-up observations have been carried out for this candidate.

\subsubsection{LRa01 E1 $4836-\mathrm{MON}-0102630623$}

Analysis of this $\mathrm{V}$-shaped transit candidate $(D=4.70 \%, P=$ 36.78 days) shows a significant depth difference between even and odd transits $(12 \sigma)$, suggesting an eclipsing binary scenario. It is therefore a low-priority candidate for which no follow-up observations have been carried out. Exo-Dat lists the spectral type of this $V=15.85 \mathrm{mag}$ star as A5 V.

\subsubsection{LRa01 E1 $5450-\mathrm{MON}-0102595916$}

The $0.22 \%$ deep transit signal in the lightcurve of this G0 IV star of brightness $V=16.38 \mathrm{mag}$ (Exo-Dat) appears to have an asymmetric shape. In addition, the transit duration of $9.23 \mathrm{~h}$ is too long for the 4.11 days transit period to be consistent with a planet. Owing to the faintness of the star and the bad transit properties the candidate has a low priority in the follow-up chain and was not observed.

\subsubsection{LRa01 E2 2185 - MON - 0102729260}

This G2 V star with $V=15.08$ mag (Exo-Dat) shows V-shaped transit signals with a depth of $0.24 \%$ and a period of 1.69 days. The candidate is already known from the CoRoT IRa01 run as IRa01 E1 1319 (Carpano et al. 2009). The transit duration of $3.57 \mathrm{~h}$ seems to be quite long for a planetary object. In addition, a secondary eclipse at phase $=0.5$ and depth differences between even and odd transits were detected. This is most likely a binary system. Follow-up observations for this candidate are not planned.

\subsubsection{LRa01 E2 2597 - CHR - 0102672065}

A 8.90 days transit signal is found in the lightcurve of this $V=14.17$ mag star. AAOmega spectroscopy shows the candidate to be a G6 III/IV star, in good agreement with the Exo-Dat classification (G5 III). FLAMES yields $T_{\mathrm{eff}}=4991 \pm 140 \mathrm{~K}$, $\log g=3.24 \pm 0.30 \mathrm{dex},[\mathrm{m} / \mathrm{H}]=-0.29 \pm 0.15 \mathrm{dex}$, and $v \sin i=$ $4.8 \pm 2.0 \mathrm{~km} \mathrm{~s}^{-1}$ (Gazzano et al. 2010). As described in Sect. 3, the deep signal ( $1.00 \%$ when normalized to the blue flux only) is seen only in the CoRoT blue channel (Fig. 4). The event is detected neither in the green nor in the red lightcurve with a $12 \sigma$ and $25 \sigma$ significance, respectively. This indicates that the candidate is with high probability a contaminating eclipsing binary (CEB).

\subsubsection{LRa01 E2 2627 - CHR - 0102757559}

According to low-resolution spectroscopy performed with AAOmega the spectral type of this $V=15.13 \mathrm{mag}$ star is F4 V, whereas Exo-Dat lists this target as a G2 V object. A V-shaped deep signal $(0.083 \%)$ is found only in the CoRoT blue channel, with a significant non-detection in the green and red lightcurves $(4 \sigma)$. This indicates a contaminating eclipsing 
binary with a period of 0.95 days. Neither photometric nor additional spectroscopic follow-up is planned.

\subsubsection{LRa01 E2 $3157-\mathrm{CHR}-0102672700$}

The object is a low-priority $0.24 \%$ deep candidate $(P=$ 1.87 days) owing to 1$) \mathrm{V}$-shaped transit curve; 2) differences in the transit depths at a $5 \sigma$ significance; and 3) the transit is only seen in the red channel with a $\sim 3 \sigma$ significant no-detection in the blue and green colors, suggesting the presence a nearby contaminating eclipsing binary. Exo-Dat lists the spectral type of this $V=14.86$ mag star as G0 IV. No follow-up observations have been carried out for this star because a CEB scenario is suspected.

\subsubsection{LRa01 E2 4494 - MON - 0102587927}

A shallow transit $(0.13 \%)$ signal with a period of about 2 days is found in the CoRoT lightcurve of this faint ( $V=16.07 \mathrm{mag})$ target. Exo-Dat lists the spectral type as K3 V. The apparent transit shape is asymmetric and the transit duration $(2.81 \mathrm{~h})$ is quite long for the orbital period. No follow-up observations have been performed on this candidate.

\subsubsection{LRa01 E2 4910 - MON - 0102780627}

This is a candidate around a star of brightness $V=15.36 \mathrm{mag}$ listed as an F8 dwarf in Exo-Dat. It has been also detected in the IRa01 run (IRa01 E1 1531) and reported as a planetary candidate in Carpano et al. (2009). Observations with AAOmega classify this candidate as an F7/8 dwarf star, in really good agreement with Exo-Dat. The transit is $0.87 \%$ deep and appears every 2.38 days. IAC 80 observations confirms the transit signal is on target. Owing to secondary faint eclipses at phase 0.5 that were recently detected in the lightcurve, it is suspected to be a binary. No additional follow-up is planned.

\subsubsection{LRa01 E2 5194 - MON - 0102604000}

This candidate is suspected to be a binary. The $2.79 \mathrm{~h}$ duration of the $0.68 \%$ deep transit is too long for a transiting planet with an orbit period of 1.25 days around a K2V star (Exo-Dat). No spectroscopic follow-up observations have been made owing to the faintness of the star ( $V=16.09 \mathrm{mag})$ and the lack of groundbased confirmation that the transit is on target.

\subsection{False alarms}

Sometimes, CoRoT lightcurves are affected by instrumental effects (e.g., jumps, glitches), which make the transit detection uncertain.

\subsubsection{LRa01 E1 $2960-$ CHR - 0102613782}

There appears to be a $0.17 \%$ deep transit with a 13.03 days period in this lightcurve of a $V=14.40 \mathrm{mag}$ F8 IV star (Exo-Dat). This signal was only detected in the CoRoT red channel, which is affected by instrumental effects (jumps). If real and on target, the transit should be visible in the green and blue channel as well. Therefore, it is suspected to be a false alarm.

\subsubsection{LRa01 E2 $3389-\mathrm{CHR}-0102674894$}

This shallow transit signal $(D=0.08 \%, P=7.03$ days $)$ is found in the lightcurve of a faint $(V=15.65 \mathrm{mag})$ G0 III star (Exo-Dat), but the lightcurve is disturbed by instrumental effects (jumps). It is suspected to be a false alarm.

\subsubsection{LRa01 E2 $3612-$ MON - 0102577194}

A $0.34 \%$ deep transit signal with a period of 38.24 days is found in the lightcurve of this faint object ( $V=16.01 \mathrm{mag})$, listed as a G0 IV star in Exo-Dat. The shape of the transit is asymmetric and the CoRoT data suffer from glitches and jumps. This is likely a false alarm.

\subsection{X-case candidates}

The following stars are objects that might be planetary candidates if the spectral type of the target were considerable different than listed in Exo-Dat.

\subsubsection{LRa01 E2 $0928-\mathrm{MON}-0102664130$}

The target star of this $2.60 \%$ deep transit candidate is according to Exo-Dat an A5 IV star, implying a stellar radius of about $2 R_{\odot}$ (Cox 2000). If true, the V-shaped signal, occurring every 49.9 days, cannot be caused by a planet. But the true spectral type of a star can differ significantly from the one given in Exo-Dat. Therefore the candidate was not completely discarded as a binary pending the confirmation of the spectral type. Unfortunately, the star is relatively faint ( $V=15.62 \mathrm{mag}$ ), making a rapid spectral type classification difficult.

\subsubsection{LRa01 E2 $5678-\mathrm{MON}-0102613411$}

Another deep candidate that might be a planetary candidate if the true stellar radius is smaller than listed in Exo-Dat (i.e., $R_{*} \approx$ $1.1 R_{\odot}$ for an F8 V star). The transit is $4.33 \%$ deep and occurs every 18.76 days. Unfortunately, the star is relatively faint $(V=$ $15.91 \mathrm{mag}$ ), making it a difficult target for a rapid spectral type classification.

\subsection{Mono-transits}

\subsubsection{LRa01 E1 $2765-\mathrm{MON}-0102647266$}

A mono-transit candidate is found with $D=8.30 \%$ at epoch $\mathrm{HJD}=2454465.434$ in the light curve of an A5 V star of magnitude $V=14.52 \mathrm{mag}$ (Exo-Dat).

\subsubsection{LRa01 E1 $4785-\mathrm{CHR}-0102709133$}

This single transit $(D=9.70 \%$ at HJD $=2454419.798)$ is more prominent in the blue channel and there might be a secondary smaller eclipse at HJD $=2454524.348$ with a depth of $\sim 1 \%$. This candidate is probably a contaminating eclipsing binary with a period of $\sim 209$ days. The target star is listed in Exo-Dat as a K2 V star with magnitude $V=15.50$ mag.

\subsubsection{LRa01 E2 $1113-\mathrm{CHR}-0102574444$}

At epoch HJD $=2454422.342$ a $5.40 \%$ deep transit can be found in the lightcurve of this star. The transit signal is deeper in the blue channel. Probably the transiting object does not orbit 


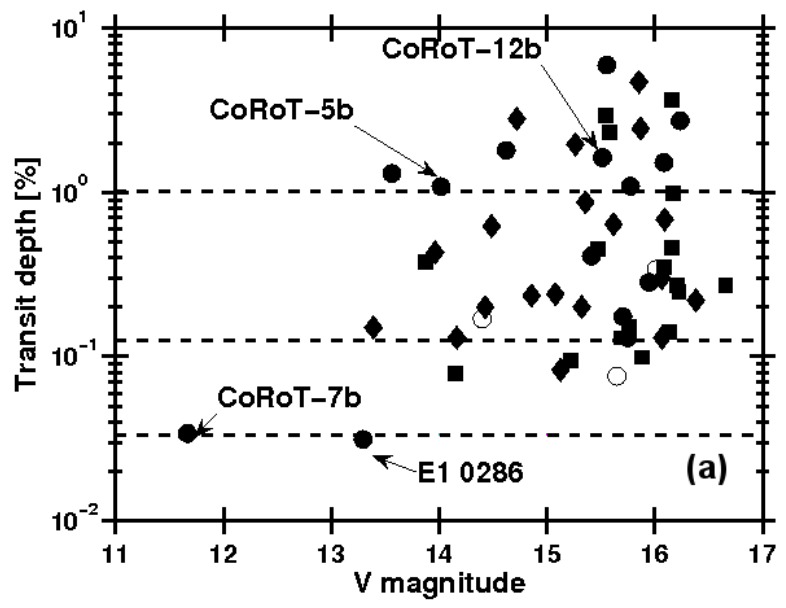

Fig. 13. Transit depth $(D)$ versus $V$ magnitude of the candidates found in LRa01 by the detection group after exploiting the full length of the lightcurves. Filled circles are good planetary candidates, squares are low-priority candidates, diamonds are suspected binaries, and open circles are suspected false alarms. Arrows mark the three transiting planets found in the LRa01 run: CoRoT-5b, CoRoT-7b and CoRoT-12b, and the suspected planet LRa01 E1 0286. The horizontal dashed lines represent (from top to bottom) the expected signal produced by a Jupiter-size planet, a Neptune-size planet and a 2 Earth radii planet around a solarlike star, respectively.

this F8 IV star of brightness $V=14.14$ mag (Exo-Dat). It is a suspected contaminating eclipsing binary.

\subsubsection{LRa01 E2 $2368-\mathrm{MON}-0102582649$}

The lightcurve of this target shows a single deep eclipse $(D=$ $17.80 \%$ ) at epoch 2454510.992 HJD. The $V=14.98$ mag object is listed in Exo-Dat as an F6 V star.

\subsubsection{LRa01 E2 2744 - MON - 0102586624}

At epoch HJD $=2454473.174$ a $15.75 \%$ deep single eclipse is visible in the lightcurve of this target. The $V=15.11$ mag object is listed in Exo-Dat as an A5 IV star.

\section{Discussion}

CoRoT-7b (Léger et al. 2009) and the candidate LRa01 E1 0286 prove the capability of CoRoT for discovering Super-Earths around main-sequence stars. Although the latter candidate is maybe not a Super-Earth, the depth of the detected transit is comparable to the signal expected from a bona fide Super-Earth around a solar-like star. Therefore, it is concluded from Fig. 13 that CoRoT is capable of detecting Super-Earths with periods in the range of one to four days around stars of apparent magnitude $V \leq 13.3 \mathrm{mag}$. However, most of the target stars are fainter than this limit (Fig. 1). The CoRoT-7b case was favorable for the terrestrial-size planet detection. Indeed, the planet orbits a star of magnitude $V=11.65 \mathrm{mag}$ in less than one day. CoRoT-7 is one of the brightest objects in the LRa01 star field. The follow-up for the candidate LRa01 E1 0286 proved to be much more difficult although the star of magnitude $V=13.30 \mathrm{mag}$ is still quite bright compared to others in the field.

Compared to previous CoRoT runs (i.e., IRa01, SRc01, and LRc01), more and weaker candidates have been found in the LRa01 field (Figs. 14 and 15). This was expected, because

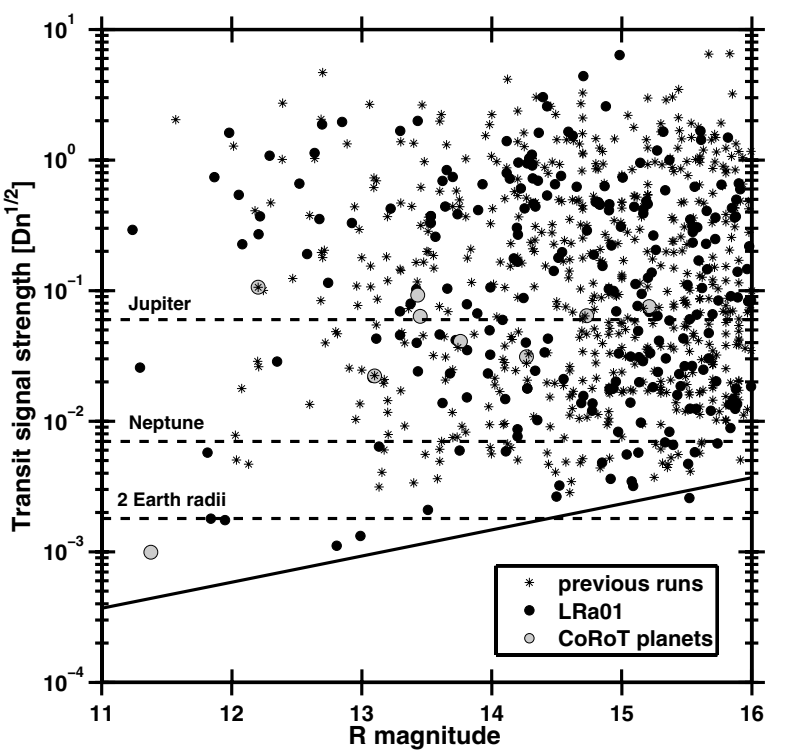

Fig. 14. Transit signal strength versus $r^{\prime}$ magnitude of detected planetary and binary candidates for the previous IRa01, SRc01, LRc01 CoRoT runs (asterisks), LRa01 run (filled circles), and first seven CoRoT-planets (open circles). $D$ is the transit depth and $n$ is the number of points in a transit event. The horizontal dashed lines represent (from top to bottom) the expected signal produced by a Jupiter-size planet, a Neptune-size planet, and a two-Earth radii planet around a solar-like star, respectively. Transit candidates and binaries were combined in this example to have a better statistical basis of the detection efficiency. The solid line represents the photon noise.

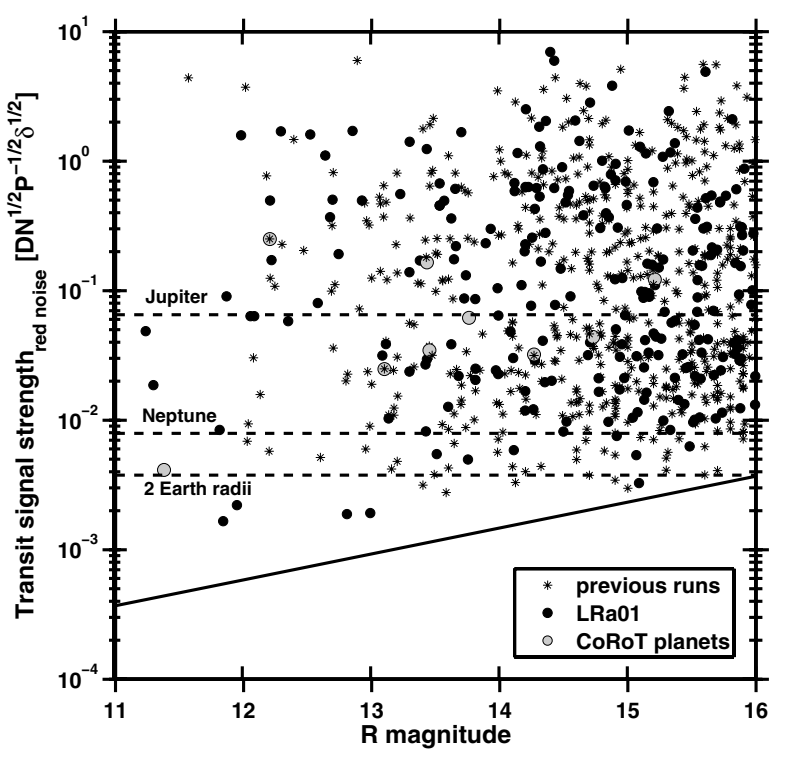

Fig. 15. Transit signal strength in the presence of red noise versus $r^{\prime}$ magnitude for the detected and binaries candidates in the previous IRa01, SRc01, LRc01 CoRoT runs (asterisks), LRa01 run (filled circles), and the first seven CoRoT-planets (open circles). $D$ is the transit depth, $N$ is the number of data points in a lightcurve, $P$ is the transit period, and $\delta=512 \mathrm{~s}$ is the sampling interval. The horizontal dashed lines represent (from top to bottom) the expected signal produced by a Jupiter-size planet with a three-day orbit period, a Neptune-size planet with a three-day orbit period, and a two-Earth radii planet with a oneday orbit period around a solar-like star, respectively. The solid line represents the photon noise.

LRa01 covers a longer time period than SRc01 and IRa01 ( $\approx 25$ days for SRc01 and $\approx 90$ days for IRa01). The lack of 
Table 3. Number of $V<13$ mag dwarf stars observed by CoRoT in the IRa01, LRc01, and LRa01 fields, as retrieved from Exo-Dat.

\begin{tabular}{lc}
\hline \hline $\begin{array}{l}\text { CoRoT } \\
\text { run }\end{array}$ & $\begin{array}{c}\text { Number of dwarf stars } \\
\text { with } V<13 \mathrm{mag}\end{array}$ \\
\hline IRa01 & 308 \\
LRc01 & 111 \\
LRa01 & 438 \\
\hline
\end{tabular}

small transit candidates around bright stars in the LRc01 star field is explained by the unfavorable stellar population properties of this particular star field for the search of extrasolar planets. According to Exo-Dat, only $\sim 37.3 \%$ of all stars in LRc01 were identified as dwarfs. Furthermore, LRc01 contains in total less stars with apparent magnitude $V<13$ mag. On the other hand, $\sim 50 \%$ of the stars observed by CoRoT in the LRa01 field are main-sequence stars. Overall, LRa01 contains more bright mainsequence stars with apparent magnitude $V<13$ mag than previous long runs (Sect. 2 and Table 3). Planets around such stars are easier to detect in the photometric data and easier to observe by ground-based telescopes. From the perspective of the types of false positives and bona fide transiting planets identified in LRa01 with respect to IRa01 and LRc01, we found that the rates of discovered planets, spectroscopically confirmed eclipsing binary systems, $\mathrm{CEB}$, and blends is comparable between the three runs.

The lack of confirmed Neptune-size planets is probably not caused by limitations in the CoRoT follow-up or detection chain. Although the follow-up is constrained by the limited observation time as pointed out by Moutou et al. (2009), CoRoT was able to find at least one Super-Earth and other planet search programs yield similar result. Short-period Neptune-size planets may be very rare objects, as tentatively pointed out 2005 by Mazeh et al. (2005) and as seems to be confirmed by the distribution of Kepler planetary candidates (Borucki et al. 2011). Such planets may evaporate faster than more massive gas giants because their low surface gravity is not able to maintain the irradiated atmosphere (Southworth et al. 2007). Davis \& Wheatley (2009) provide additional evidence for a lost population of shortperiod Neptune planets. Almenara et al. (2009), after analysis of three CoRoT fields, further support the conclusion that the lack of confirmed Neptune planets in the CoRoT fields is not caused by observational limitations.

\section{Summary}

CoRoT observed the LRa01 star field continuously for 130 days and collected 11408 lightcurves. There are 7470 chromatic photometric data-sets and 3938 monochromatic data-sets. The CoRoT detection group performed a full in-depth analysis of the lightcurves. Two hundred forty-two lightcurves $(2.1 \%$ of all lightcurves) contain a transit signal. One hundred ninety-one of these ( $79 \%$ of the candidates or $1.7 \%$ of all stars in the field) were identified as binaries based on photometric analysis only (Table 7), including five mono-transit objects (Sect. 6.7).

Fifty-one signals were classified as planetary candidates and proposed for observational follow-up with different priorities based on the photometric analysis. Thus in about $0.5 \%$ of all CoRoT targets a signal was detected that might originate from a planetary transit. In addition, three candidates were discarded as likely false alarms based on photometric analysis (Sect. 6.5). Five mono-transits were detected with depths compatible with eclipsing binary systems (Sect. 6.7 and Table 7). Two candidates were classified as potential planetary candidates or "X-cases" (Sect. 6.6), if the stellar radius of the target star is significantly smaller than listed in the Exo-Dat database. None of the false candidates and X-cases were followed-up but they are included for completeness. See also Table 5.

Of the fifty-one candidates, thirty-seven (73\% of all candidates) are "good" planetary candidates based on photometric analysis only (Sects. 6.1-6.3). Thirty-two of the "good" candidates have been followed-up and the nature of twenty-two objects has been solved. Four candidates (about $8 \%$ of all candidates) have been confirmed as transiting planets (Table 2): CoRoT-7b (Léger et al. 2009), CoRoT-5b (Rauer et al. 2009), CoRoT-12b (Gillon et al. 2010), and the recently confirmed hotJupiter LRa01 E2 5277 (CoRoT-21b, Pätzold et al., in prep.). Another two non-transiting planets were detected by RV measurements only: CoRoT-7c (Queloz et al. 2009) and CoRoT-7d (Hatzes et al. 2010). Another candidate, LRa01 E1 0286 might be a planetary object in a binary system but is unconfirmed yet. Eighteen objects ( $49 \%$ of the good candidates) were identified as non-planetary objects. Six are contaminating eclipsing binaries (CEBs) and two are blends (i.e., LRa01 E1,1123 and LRa01 E2 5184). Six candidates are spectroscopically resolved as stellar binaries (SB). Four candidates are stellar companions around early-type stars.

According to the lightcurve analysis only, fourteen candidates (27\% of all candidates; Sect. 6.4) have low priorities because of one or more characteristics hinting at a non-planetary scenario: out-of-transit variations, depth differences between even and odd transits, depth differences in the three color channels, and very shallow secondary eclipse. Four of these were followed-up but the observations are not conclusive.

The follow-up for most of the LRa01 candidates is now concluded. Only LRa01 E1 0286, LRa01 E1 2101, LRa01 E1 4667, and LRa01 E2 3156 are still under investigation.

Acknowledgements. We thank the anonymous referee for her/his careful reading, useful comments, and suggestions, which helped to improve the manuscript. The German CoRoT Team (University of Cologne and TLS) acknowledges Deutsches Zentrum für Luft- und Raumfahrt (DLR) grants 50 QM 1004, 50 OW 0204, 50 OW 0603, and 50 QP 07011. The team at the IAC acknowledges support by grants ESP2007-65480-C02-02 and AYA2010-20982-C02-02 of the Spanish Ministerio de Ciencia e Innovación. Partly based on observations carried-out at the European Southern Observatory (ESO), La Silla and Paranal (Chile), under observing programs numbers 080.D-0151, 081.C-0388, 081.C0413, 083.C-0186, and 282.C-5015. The authors are grateful to the staff at ESO La Silla and ESO Paranal Observatories for their support and contribution to the success of the HARPS, UVES, NACO, and CRIRES observing runs. Parts of the data presented herein were also obtained at the W. M. Keck Observatory from telescope time allocated to the National Aeronautics and Space Administration through the agency's scientific partnership with the California Institute of Technology and the University of California. The Observatory was made possible by the generous financial support of the W. M. Keck Foundation. The authors wish to recognize and acknowledge the very significant cultural role and reverence that the summit of Mauna Kea has always had within the indigenous Hawaiian community. We are most fortunate to have the opportunity to conduct observations from this mountain. The present manuscript is also based on observations performed with a) the IAC 80 telescope operated by the Instituto de Astrofísica de Tenerife at the Observatorio del Teide. We thank its observing staff; b) the SOPHIE spectrograph at the Observatoire de Haute-Provence, France, under observing programs PNP.08A.MOUT, PNP.09A.MOUT, and PNP.10A.MOUT; c) the FIES spectrograph at the Nordic Optical Telescope (observing program P42-261), operated on the island of La Palma jointly by Denmark, Finland, Iceland, Norway, and Sweden, in the Spanish Observatorio del Roque de los Muchachos of the Instituto de Astrofisica de Canarias; d) the AAOmega multi-object facilities at the Anglo-Australian Telescope operated at Siding Spring Observatory by the Anglo-Australian Observatory (observing programs $07 \mathrm{~B} / 040$ and $08 \mathrm{~B} / 003$ ); e) the Sandiford spectrograph at the $2.1 \mathrm{~m}$ Otto Struve telescope at McDonald Observatory of the University of Texas at Austin; f) the MOnitoring NEtwork of Telescopes (MONET), funded by the "Astronomie \& Interne" program of the Alfried Krupp von Bohlen und Halbach Foundation, Essen, and operated by the Georg-August-Universität Göttingen, 
the McDonald Observatory of the University of Texas at Austin, and the South African Astronomical Observatory. The CoRoT/Exoplanet catalogue (Exo-Dat) was made possible by observations collected for years at the Isaac Newton Telescope (INT), operated on the island of La Palma by the Isaac Newton group in the Spanish Observatorio del Roque de Los Muchachos of the Instituto de Astrofísica de Canarias.

\section{References}

Alapini, A., \& Aigrain, S. 2008, in IAU Symp. 249, ed. Y.-S. Sun, S. Ferraz-Mello \& J.-L. Zhou, 89

Almenara, J. M., Deeg, H. J., Aigrain, S., et al. 2009, A\&A, 506, 337

Alonso, R., Deeg, H. J., Brown, T. M., \& Belmonte, J. A. 2004, Astron. Notes, 325,594

Auvergne, M., Bodin, P., Boisnard, L., et al. 2009, A\&A, 506, 411

Batalha, N. M., Borucki, W. J., Bryson, S. T., et al. 2011, ApJ, 729, 27

Boisse, I., Bouchy, F., Hebrard, G., et al. 2011, A\&A, 528, A4

Bordé, P., Fressin, F., Ollivier, M., Léger, A., \& Rouan, D. 2007, in Transiting Extrapolar Planets Workshop, ed. C. Afonso, D. Weldrake \& T. Henning, ASP Conf. Ser., 366, 145

Borucki, W. J., Koch, D. G., Basri, G., et al. 2011, ApJ, 736, 19

Brown, T. M. 2003, ApJ, 593, L125

Bruntt, H., Deleuil, M., Fridlund, M., et al. 2010, A\&A, 519, A51

Cabrera, J., Fridlund, M., Ollivier, M., et al. 2009, A\&A, 506, 501

Carpano, S., \& Fridlund, M. 2008, A\&A, 485, 607

Carpano, S., Cabrera, J., Alonso, R., et al. 2009, A\&A, 506, 491

Cox, A. N. 2000, Allen's astrophysical quantities, ed. A. N. Cox (New York: AIP Press; Springer)

Cutri, R. M., Skrutskie, M. F., van Dyk, S., et al. 2003, 2MASS All Sky Catalog of point sources, ed. Cutri et al., NASA/IPAC Infrared Science Archive

Davis, T. A., \& Wheatley, P. J. 2009, MNRAS, 396, 1012

Debosscher, J., Sarro, L. M., López, M., et al. 2009, A\&A, 506, 519

Deeg, H. J., Gillon, M., Shporer, A., et al. 2009, A\&A, 506, 343

Deleuil, M., Moutou, C., Deeg, H. J., et al. 2006, in ESA SP. 1306, ed. M. Fridlund, A. Baglin, J. Lochard \& L. Conroy, 341

Deleuil, M., Deeg, H. J., Alonso, R., et al. 2008, A\&A, 491, 889

Deleuil, M., Meunier, J. C., Moutou, C., et al. 2009, AJ, 138, 649

Drummond, R., Lapeyrere, V., Auvergne, M., et al. 2008, A\&A, 487, 1209

Ferraz-Mello, S., Tadeu dos Santos, M., Beauge, C., Michtchenko, T. A., \& Rodriguez, A. 2011, A\&A, 531, A161

Gandolfi, D., Alcalá, J. M., Leccia, S., et al. 2008, ApJ, 687, 1303

Gandolfi, D., Hébrard, G., Alonso, R., et al. 2010, A\&A, 524, A55

Gazzano, J.-C., de Laverny, P., Deleuil, M., et al. 2010, A\&A, 523, A91

Gillon, M., Hatzes, A., Csizmadia, S., et al. 2010, A\&A, 520, A97

Gondoin, P., Fridlund, M., Goupil, M. J., et al. 2009, in AIP Conf. Ser. 1094, ed. E. Stempels, 864

Günther, E. W., Gandolfi, D., Sebastian, D., Deleuil, M., \& Moutou, C. 2012, A\&A, submitted

Hatzes, A. P., Dvorak, R., Wuchterl, G., et al. 2010, A\&A, 520, A93

Hatzes, A. P., Fridlund, M., Nachmani, G., et al. 2011, ApJ, 743, 75

Hekker, S., Kallinger, T., Baudin, F., et al. 2009, A\&A, 506, 465

Kabath, P., Eigmüller, P., Erikson, A., et al. 2008, AJ, 136, 654

Klement, R. J., Bailer-Jones, C. A. L., Fuchs, B., Rix, H.-W., \& Smith, K. W. 2011, ApJ, 726, 103

Léger, A., Rouan, D., Schneider, J., et al. 2009, A\&A, 506, 287

Llebaria, A., \& Guterman, P. 2006, in ESA SP. 1306, ed. M. Fridlund, A. Baglin, J. Lochard, \& L. Conroy, 293

Loeillet, B., Bouchy, F., Deleuil, M., et al. 2008, A\&A, 479, 865

Mazeh, T., Zucker, S., \& Pont, F. 2005, MNRAS, 356, 955

Meunier, J.-C., Deleuil, M., Moutou, C., et al. 2007, in Astronomical Data Analysis Software and Systems XVI, ed. R. A. Shaw, F. Hill, \& D. J. Bell, ASP Conf. Ser., 376, 339

Michel, E., Baglin, A., Weiss, W. W., et al. 2008, Commun. Asteroseismol., 156, 73

Moutou, C., Pont, F., Barge, P., et al. 2005, A\&A, 437, 355

Moutou, C., Aigrain, S., Almenara, J., et al. 2007, in Transiting Extrapolar Planets Workshop, ed. C. Afonso, D. Weldrake, \& T. Henning, ASP Conf. Ser., 366, 127

Moutou, C., Pont, F., Bouchy, F., et al. 2009, A\&A, 506, 321

Pinheiro da Silva, L., Rolland, G., Lapeyrere, V., \& Auvergne, M. 2008, MNRAS, 384, 1337

Pont, F., Aigrain, S., \& Zucker, S. 2011, MNRAS, 411, 1953

Queloz, D., Bouchy, F., Moutou, C., et al. 2009, A\&A, 506, 303

Rauer, H., Queloz, D., Csizmadia, S., et al. 2009, A\&A, 506, 281

Régulo, C., Almenara, J. M., Alonso, R., Deeg, H., \& Roca Cortés, T. 2007, A\&A, 467, 1345
Renner, S., Rauer, H., Erikson, A., et al. 2008, A\&A, 492, 617

Sebastian, D., Günther, E. W., Schaffenroth, V., et al. 2012, A\&A, submitted

Southworth, J., Wheatley, P. J., \& Sams, G. 2007, MNRAS, 379, L11

Surace, C., Alonso, R., Barge, P., et al. 2008, in SPIE Conf. Ser., 7019

1 Rheinisches Institut für Umweltforschung, Abteilung Planetenforschung, an der Universität zu Köln, Aachener Strasse 209, 50931 Köln, Germany e-mail: 1carone@uni-koeln.de

2 Research and Scientific Support Department, ESTEC/ESA, PO Box 299, 2200 AG Noordwijk, The Netherlands e-mail: davide.gandolfi@esa.int

3 Thüringer Landessternwarte, Sternwarte 5, 07778 Tautenburg, Germany

${ }^{4}$ Institute of Planetary Research, German Aerospace Center, Rutherfordstrasse 2, 12489 Berlin, Germany

5 LUTH, Observatoire de Paris, CNRS, Université Paris Diderot, 5 place Jules Janssen, 92195 Meudon, France

6 Instituto de Astrofísica de Canarias, 38205 La Laguna, Tenerife, Spain

7 Departamento de Astrofísica, Universidad de La Laguna, $38206 \mathrm{La}$ Laguna, Tenerife, Spain

8 Space Research Institute, Austrian Academy of Science, Schmiedlstr. 6, 8042 Graz, Austria

9 Oxford Astrophysics, Denys Wilkinson Building, Keble Road, Oxford OX1 3RH, UK

10 Observatoire de l'Université de Genève, 51 Chemin des Maillettes, 1290 Sauverny, Switzerland

11 School of Physics, University of Exeter, Stocker Road, Exeter EX4 4QL, UK

12 Laboratoire d'Astrophysique de Marseille, CNRS \& University of Provence, 38 rue Frédéric Joliot-Curie, 13388 Marseille Cedex 13, France

13 LESIA, Observatoire de Paris, Place Jules Janssen, 92195 Meudon Cedex, France

14 Institut d'Astrophysique Spatiale, Université Paris XI, 91405 Orsay, France

15 Institut d'Astrophysique de Paris, UMR7095 CNRS, Université Pierre \& Marie Curie, 98bis boulevard Arago, 75014 Paris, France

16 Observatoire de Haute Provence, 04670 Saint Michel l'Observatoire, France

17 McDonald Observatory, University of Texas at Austin, Austin, TX 78712, USA

18 Institut für Astrophysik, Georg-August-Universität, FriedrichHund-Platz 1, 37077 Göttingen, Germany

19 University of Vienna, Institute of Astronomy, Türkenschanzstrasse 17, 1180 Vienna, Austria

20 IAG, University of São Paulo, Brasil

21 Université de Nice-Sophia Antipolis, CNRS UMR 6202, Observatoire de la Côte d'Azur, BP 4229, 06304 Nice Cedex 4, France

${ }^{22}$ University of Liège, Allée du 6 août 17, Sart Tilman, Liège 1, Belgium

23 European Southern Observatory, Alonso de Córdova 3107, Casilla 19001, Santiago de Chile, Chile

${ }^{24}$ School of Physics and Astronomy, Raymond and Beverly Sackler Faculty of Exact Sciences, Tel Aviv University, Tel Aviv, Israel

25 Departamento de Astronomía y Astrofísica, Pontificia Universidad Católica de Chile, Casilla 306, Santiago 22, Chile

${ }^{26}$ Center for Astronomy and Astrophysics, TU Berlin, Hardenbergstr. 36, 10623 Berlin, Germany

27 Laboratoire d'Astronomie de Lille, Université de Lille 1, 1 impasse de l'Observatoire, 59000 Lille, France

28 Institut de Mécanique Céleste et de Calcul des Ephémérides, UMR 8028 du CNRS, 77 avenue Denfert-Rochereau, 75014 Paris, France

29 Observatório Nacional, Rio de Janeiro, Brazil

30 Las Cumbres Observatory Global Telescope Network, Inc., 6740 Cortona Drive, Suite 102, Santa Barbara, California 93117, USA 
Table 4. Radial velocity measurements of the LRa01 planetary candidates.

\begin{tabular}{|c|c|c|c|}
\hline $\begin{array}{c}\text { BJD } \\
\text { (days] }\end{array}$ & $\begin{array}{c}\mathrm{RV} \\
{\left[\mathrm{km} \mathrm{s}^{-1}\right]}\end{array}$ & $\begin{array}{l}\sigma \mathrm{RV} \\
{\left[\mathrm{km} \mathrm{s}^{-1}\right]}\end{array}$ & Instrument \\
\hline \multicolumn{4}{|l|}{ LRa01 E1 0544} \\
\hline 2454766.69555 & 16.879 & 0.038 & SOPHIE \\
\hline 2454767.64784 & 16.780 & 0.056 & SOPHIE \\
\hline \multicolumn{4}{|l|}{ LRa01 E1 0561} \\
\hline 2454515.34284 & 43.753 & 0.161 & SOPHIE \\
\hline 2454526.33924 & -8.432 & 0.132 & SOPHIE \\
\hline \multicolumn{4}{|l|}{ LRa01 E1 5015} \\
\hline 2455162.72198 & 62.831 & 0.246 & HARPS \\
\hline 2455167.81154 & 92.556 & 0.251 & HARPS \\
\hline \multicolumn{4}{|l|}{ LRa01 E2 1123} \\
\hline 2454503.65300 & -3.679 & 0.142 & CORALIE \\
\hline 2454536.58722 & -3.727 & 0.138 & CORALIE \\
\hline 2454552.50781 & -3.718 & 0.018 & HARPS \\
\hline 2454555.53324 & -3.686 & 0.020 & HARPS \\
\hline 2454556.52061 & -3.723 & 0.019 & HARPS \\
\hline 2454557.52309 & -3.729 & 0.017 & HARPS \\
\hline 2454748.86821 & -3.708 & 0.017 & HARPS \\
\hline 2454762.82555 & -3.673 & 0.015 & HARPS \\
\hline \multicolumn{4}{|c|}{ LRa01 E2 1145 = IRa01 E1 1873} \\
\hline 2454887.40723 & 25.201 & 0.073 & SOPHIE \\
\hline 2454890.36710 & 71.572 & 0.069 & SOPHIE \\
\hline \multicolumn{4}{|l|}{ LRa01 E2 2249 } \\
\hline 2454809.59849 & 36.297 & 0.017 & SOPHIE \\
\hline 2454822.54316 & 60.556 & 0.011 & SOPHIE \\
\hline \multicolumn{4}{|l|}{ LRa01 E2 5084} \\
\hline 2455151.85444 & 81.812 & 0.497 & HARPS \\
\hline 2455166.76997 & 8.308 & 0.385 & HARPS \\
\hline \multicolumn{4}{|c|}{ LRa01 E2 5184 = IRa01 E1 4108} \\
\hline 2454516.60149 & 99.250 & 0.024 & HARPS \\
\hline 2454518.64495 & 99.220 & 0.031 & HARPS \\
\hline 2454519.59227 & 99.321 & 0.032 & HARPS \\
\hline 2455152.81296 & 99.261 & 0.021 & HARPS \\
\hline \multicolumn{4}{|l|}{ LRa01 E1 0286} \\
\hline 2454883.64831 & 118.833 & 0.124 & Sandiford \\
\hline 2454885.78471 & 118.985 & 0.132 & Sandiford \\
\hline 2455584.79281 & 121.705 & 0.112 & Sandiford \\
\hline 2455591.77678 & 122.586 & 0.094 & Sandiford \\
\hline 2455141.63827 & 115.928 & 0.012 & SOPHIE \\
\hline 2455214.43238 & 118.123 & 0.014 & SOPHIE \\
\hline 2455238.37453 & 120.656 & 0.021 & SOPHIE \\
\hline 2455240.39734 & 120.891 & 0.015 & SOPHIE \\
\hline 2455242.37050 & 121.088 & 0.011 & SOPHIE \\
\hline 2455269.32548 & 124.209 & 0.010 & SOPHIE \\
\hline 2455271.30759 & 124.406 & 0.008 & SOPHIE \\
\hline 2455273.31278 & 124.586 & 0.015 & SOPHIE \\
\hline 2455615.32719 & 124.981 & 0.009 & SOPHIE \\
\hline 2455618.33486 & 125.277 & 0.009 & SOPHIE \\
\hline 2455170.88184 & -4.642 & 0.007 & HIRES \\
\hline 2455171.14966 & -4.638 & 0.007 & HIRES \\
\hline 2455222.77727 & -1.160 & 0.007 & HIRES \\
\hline 2455223.92979 & -1.052 & 0.007 & HIRES \\
\hline 2455224.78564 & -0.956 & 0.005 & HIRES \\
\hline 2455225.06690 & -0.922 & 0.005 & HIRES \\
\hline 2455609.78808 & 4.419 & 0.010 & HIRES \\
\hline 2455609.79556 & 4.421 & 0.007 & HIRES \\
\hline 2455610.94623 & 4.530 & 0.008 & HIRES \\
\hline 2455245.67304 & 121.487 & 0.003 & HARPS \\
\hline 2455247.69007 & 121.710 & 0.013 & HARPS \\
\hline 2455505.85621 & 115.387 & 0.005 & HARPS \\
\hline 2455507.79110 & 115.391 & 0.004 & HARPS \\
\hline 2455510.78882 & 115.451 & 0.006 & HARPS \\
\hline 2455512.78152 & 115.504 & 0.005 & HARPS \\
\hline 2455514.79798 & 115.575 & 0.004 & HARPS \\
\hline 2455515.78588 & 115.607 & 0.005 & HARPS \\
\hline
\end{tabular}

Table 4. continued.

\begin{tabular}{|c|c|c|c|}
\hline $\begin{array}{l}\text { BJD } \\
\text { (days] }\end{array}$ & $\begin{array}{c}\mathrm{RV} \\
{\left[\mathrm{km} \mathrm{s}^{-1}\right]}\end{array}$ & $\begin{array}{c}\sigma \mathrm{RV} \\
{\left[\mathrm{km} \mathrm{s}^{-1}\right]}\end{array}$ & Instrument \\
\hline 2455517.80895 & 115.668 & 0.003 & HARPS \\
\hline 2455521.79614 & 115.842 & 0.009 & HARPS \\
\hline 2455524.78741 & 115.995 & 0.004 & HARPS \\
\hline 2455575.71323 & 120.612 & 0.007 & HARPS \\
\hline 2455575.75009 & 120.618 & 0.004 & HARPS \\
\hline 2455577.67357 & 120.845 & 0.006 & HARPS \\
\hline 2455577.71019 & 120.844 & 0.006 & HARPS \\
\hline 2455569.68872 & 119.876 & 0.025 & FIES \\
\hline 2455581.46264 & 121.240 & 0.024 & FIES \\
\hline \multicolumn{4}{|l|}{ LRa01 E1 2101} \\
\hline 2455220.64433 & 0.907 & 0.012 & HARPS \\
\hline 2455240.58607 & 0.909 & 0.028 & HARPS \\
\hline 2455583.57129 & 0.928 & 0.013 & HARPS \\
\hline 2455583.60336 & 0.921 & 0.017 & HARPS \\
\hline 2455584.65380 & 0.903 & 0.020 & HARPS \\
\hline 2455584.68555 & 0.955 & 0.018 & HARPS \\
\hline \multicolumn{4}{|l|}{ LRa01 E1 4667} \\
\hline 2455157.81799 & 26.205 & 0.078 & HARPS \\
\hline 2455166.81523 & 26.121 & 0.056 & HARPS \\
\hline \multicolumn{4}{|l|}{ LRa01 E23156 } \\
\hline 2455157.77092 & 82.084 & 0.033 & HARPS \\
\hline 2455159.76521 & 82.118 & 0.035 & HARPS \\
\hline 2455222.00327 & 0.013 & 0.017 & HIRES \\
\hline 2455222.01408 & -0.008 & 0.019 & HIRES \\
\hline 2455222.73752 & -0.001 & 0.019 & HIRES \\
\hline 2455223.81145 & 0.000 & 0.019 & HIRES \\
\hline 2455224.00538 & 0.000 & 0.017 & HIRES \\
\hline 2455224.74151 & -0.020 & 0.024 & HIRES \\
\hline 2455610.92447 & 0.015 & 0.017 & HIRES \\
\hline
\end{tabular}


Table 5. CoRoT LRa01 planetary candidates: coordinates and transit parameters.

\begin{tabular}{|c|c|c|c|c|c|c|c|}
\hline CoRoT ID & in ID & $\begin{array}{c}\alpha(\mathrm{J} 2000) \\
{[\mathrm{deg}]}\end{array}$ & $\begin{array}{c}\delta(\mathrm{J} 2000) \\
{[\mathrm{deg}]}\end{array}$ & $\begin{array}{c}\text { Epoch (HJD) } \\
\text { [days] }\end{array}$ & $\begin{array}{l}\text { Period } \\
{[\text { Days }]}\end{array}$ & $\begin{array}{l}\text { Depth } \\
\%\end{array}$ & $\begin{array}{l}\text { Duration } \\
{[\mathrm{h}]}\end{array}$ \\
\hline $\begin{array}{l}102764809 \\
102708694 \\
102671819 \\
102725122 \\
\end{array}$ & $\begin{array}{l}\text { E1 } 1031 \\
\text { E2 } 0165 \\
\text { E2 } 3459 \\
\text { E2 } 5277 \\
\end{array}$ & $\begin{array}{l}101.27723 \\
100.95606 \\
100.76568 \\
101.05245 \\
\end{array}$ & $\begin{array}{l}0.81527 \\
-1.06303 \\
-1.29645 \\
-0.29913 \\
\end{array}$ & $\begin{array}{l}\text { Confirmed planets } \\
2454400.19885 \pm 0.00020 \\
2454398.07670 \pm 0.00150 \\
2454398.62707 \pm 0.00036 \\
2454399.02987 \pm 0.00090\end{array}$ & $\begin{array}{l}4.037896 \pm 0.000002 \\
0.853585 \pm 0.000024 \\
2.828042 \pm 0.000013 \\
2.724740 \pm 0.000140 \\
\end{array}$ & $\begin{array}{l}1.461 \\
0.034 \\
1.744 \\
0.449 \\
\end{array}$ & $\begin{array}{l}2.81 \\
1.25 \\
2.57 \\
4.76 \\
\end{array}$ \\
\hline $\begin{array}{l}102714746 \\
102597681 \\
102618931 \\
102790970 \\
102777869 \\
102692038 \\
102615551 \\
102707895 \\
102658070 \\
102755837 \\
102723949 \\
102590741 \\
102590008 \\
102667981 \\
102779966 \\
102753331 \\
102755764 \\
102582529 \\
\end{array}$ & $\begin{array}{ll}\text { E1 } & 0544 \\
\text { E1 } & 0561 \\
\text { E1 } 2890 \\
\text { E1 } 3666 \\
\text { E1 } 5015 \\
\text { E1 } 4353 \\
\text { E2 } 1123 \\
\text { E2 } 1145 \\
\text { E2 } 1897 \\
\text { E2 } 2249 \\
\text { E2 } 2481 \\
\text { E2 } 2694 \\
\text { E2 } 4129 \\
\text { E2 } 5084 \\
\text { E2 } 5184 \\
\text { E2 } 5747 \\
\text { E2 } 3739 \\
\text { E2 } 5756 \\
\end{array}$ & $\begin{array}{l}100.99094 \\
100.34489 \\
100.47033 \\
101.42390 \\
101.35044 \\
100.87058 \\
100.45001 \\
100.95144 \\
100.69371 \\
101.22621 \\
101.04563 \\
100.29606 \\
100.29088 \\
100.74543 \\
101.36197 \\
101.21195 \\
101.22575 \\
100.23665 \\
\end{array}$ & $\begin{array}{c}\text { Settle } \\
0.73759 \\
-0.18307 \\
0.81683 \\
-0.14321 \\
0.46030 \\
0.24945 \\
-0.58771 \\
-0.96872 \\
-0.34008 \\
-0.76273 \\
-1.18701 \\
-0.97935 \\
-1.37087 \\
-1.03863 \\
-1.23596 \\
-0.71480 \\
-1.50861 \\
-0.43089 \\
\end{array}$ & $\begin{array}{l}\text { cases: non-planetary objects } \\
2454398.49627 \pm 0.00001 \\
2454426.95000 \pm 0.00200 \\
2454399.29816 \pm 0.00438 \\
2454398.26940 \pm 0.00300 \\
2454405.89953 \pm 0.00006 \\
2454401.96117 \pm 0.00001 \\
2454400.84404 \pm 0.00830 \\
2454402.88326 \pm 0.00630 \\
2454402.33702 \pm 0.00008 \\
2454411.06010 \pm 0.00490 \\
2454426.74091 \pm 0.00392 \\
2454418.36090 \pm 0.00670 \\
2454398.24571 \pm 0.00470 \\
2454405.70210 \pm 0.00450 \\
2454403.17577 \pm 0.00028 \\
2454400.67866 \pm 0.00740 \\
2454426.27433 \pm 0.00200 \\
2454399.72057 \pm 0.00029 \\
\end{array}$ & $\begin{array}{c}2.75138 \pm 0.00001 \\
20.82000 \pm 0.01000 \\
2.42955 \pm 0.00026 \\
1.55028 \pm 0.00006 \\
13.68737 \pm 0.00006 \\
5.23157 \pm 0.00002 \\
3.87707 \pm 0.00004 \\
5.78390 \pm 0.00048 \\
4.66957 \pm 0.00008 \\
27.92620 \pm 0.00180 \\
51.75900 \pm 0.00590 \\
30.39480 \pm 0.00160 \\
1.94250 \pm 0.00010 \\
9.91904 \pm 0.00013 \\
7.36827 \pm 0.00028 \\
19.75240 \pm 0.00190 \\
61.48000 \pm 0.01000 \\
15.84215 \pm 0.00029 \\
\end{array}$ & $\begin{array}{l}0.150 \\
0.700 \\
0.286 \\
0.453 \\
0.995 \\
1.090 \\
1.800 \\
0.430 \\
2.800 \\
0.380 \\
1.200 \\
1.302 \\
0.175 \\
0.284 \\
0.410 \\
3.640 \\
2.930 \\
2.722 \\
\end{array}$ & $\begin{array}{c}2.59 \\
3.60 \\
1.70 \\
2.75 \\
10.10 \\
4.37 \\
1.08 \\
9.45 \\
3.65 \\
15.63 \\
0.80 \\
8.76 \\
2.02 \\
2.55 \\
2.68 \\
14.13 \\
6.97 \\
4.10 \\
\end{array}$ \\
\hline $\begin{array}{l}102742060 \\
102568803 \\
102698887 \\
102754163 \\
102634864 \\
102782651 \\
102617334 \\
102588881 \\
102703155 \\
102751316 \\
102666452 \\
102670085 \\
102716818 \\
102765275 \\
102580137\end{array}$ & $\begin{array}{ll}\text { E1 } & 0286 \\
\text { E1 } & 2101 \\
\text { E1 } & 2240 \\
\text { E1 } & 3216 \\
\text { E1 } & 3221 \\
\text { E1 } & 4423 \\
\text { E1 } & 4594 \\
\text { E1 } & 4667 \\
\text { E1 } & 4719 \\
\text { E1 } & 4820 \\
\text { E1 } & 5320 \\
\text { E1 } & 5536 \\
\text { E2 } & 3156 \\
\text { E2 } & 3619 \\
\text { E2 } & 4519\end{array}$ & $\begin{array}{l}101.14948 \\
100.13809 \\
100.90557 \\
101.21685 \\
100.56930 \\
101.37645 \\
100.46082 \\
100.28253 \\
100.92678 \\
101.20066 \\
100.73750 \\
100.75661 \\
101.00302 \\
101.27973 \\
100.21993\end{array}$ & $\begin{array}{c}\text { Unset } \\
0.00790 \\
0.28304 \\
0.28097 \\
-0.06793 \\
0.83454 \\
0.99833 \\
-0.16461 \\
0.57086 \\
0.82986 \\
0.69970 \\
1.00772 \\
0.53083 \\
-0.43469 \\
-0.67173 \\
-0.45953\end{array}$ & $\begin{array}{l}\text { ed good planetary candidates } \\
2454398.70250 \pm 0.01200 \\
2454464.06660 \pm 0.00680 \\
2454463.10470 \pm 0.00700 \\
2454464.77560 \pm 0.00740 \\
2454425.32970 \pm 0.00210 \\
2454463.41470 \pm 0.00930 \\
2454398.58412 \pm 0.01300 \\
2454409.36160 \pm 0.00340 \\
2454463.08060 \pm 0.00020 \\
2454399.51281 \pm 0.00150 \\
2454463.64950 \pm 0.00840 \\
2454398.54805 \pm 0.00220 \\
2454398.65000 \pm 0.00500 \\
2454431.97000 \pm 0.01000 \\
2454462.91720 \pm 0.06500\end{array}$ & $\begin{array}{c}3.60207 \pm 0.00005 \\
2.71839 \pm 0.00051 \\
2.03187 \pm 0.00047 \\
3.11011 \pm 0.00054 \\
32.32770 \pm 0.00080 \\
1.86570 \pm 0.00047 \\
5.48907 \pm 0.00091 \\
27.28810 \pm 0.00120 \\
1.25939 \pm 0.00020 \\
1.61152 \pm 0.00003 \\
1.96423 \pm 0.00480 \\
0.89910 \pm 0.00010 \\
1.47040 \pm 0.00020 \\
50.90780 \pm 0.00300 \\
2.37289 \pm 0.00042\end{array}$ & $\begin{array}{l}0.031 \\
0.079 \\
0.094 \\
0.130 \\
2.330 \\
0.250 \\
0.270 \\
1.520 \\
0.099 \\
0.463 \\
0.140 \\
0.270 \\
0.153 \\
5.960 \\
0.130\end{array}$ & $\begin{array}{l}1.80 \\
1.00 \\
2.10 \\
4.00 \\
4.10 \\
3.50 \\
6.62 \\
2.64 \\
0.97 \\
1.43 \\
3.30 \\
1.54 \\
2.01 \\
7.00 \\
1.87\end{array}$ \\
\hline $\begin{array}{l}102625386 \\
102617210 \\
102732757 \\
102626872 \\
102620061 \\
102630623 \\
102595916 \\
102729260 \\
102672065 \\
102757559 \\
102672700 \\
102587927 \\
102780627 \\
102604000 \\
\end{array}$ & $\begin{array}{l}\text { E1 } 2970 \\
\text { E1 } 3617 \\
\text { E1 } 3674 \\
\text { E1 } 4272 \\
\text { E1 } 4777 \\
\text { E1 } 4836 \\
\text { E1 } 5450 \\
\text { E2 } 2185 \\
\text { E2 } 2597 \\
\text { E2 } 2627 \\
\text { E2 } 3157 \\
\text { E2 } 4494 \\
\text { E2 } 4910 \\
\text { E2 } 5194\end{array}$ & $\begin{array}{l}100.51011 \\
100.46010 \\
101.09706 \\
100.51949 \\
100.47743 \\
100.54263 \\
100.33219 \\
101.07759 \\
100.76698 \\
101.23591 \\
100.77027 \\
100.27591 \\
101.36562 \\
100.38392 \\
\end{array}$ & $\begin{array}{c}\text { Unsettlec } \\
0.04896 \\
0.57616 \\
-0.06585 \\
1.04640 \\
0.45610 \\
0.47369 \\
0.07959 \\
-1.48648 \\
-1.41680 \\
-1.34969 \\
-0.36156 \\
-1.54206 \\
-0.58463 \\
-0.79700 \\
\end{array}$ & $\begin{array}{l}\text { low-priority planetary candide } \\
2454400.16994 \pm 0.00170 \\
2454463.42320 \pm 0.00910 \\
2454398.39349 \pm 0.00370 \\
2454398.57854 \pm 0.00200 \\
2454399.26041 \pm 0.00170 \\
2454405.73688 \pm 0.00220 \\
2454400.05000 \pm 0.03200 \\
2454399.74256 \pm 0.00017 \\
2454460.62310 \pm 0.00070 \\
2454463.26830 \pm 0.01060 \\
2454398.42115 \pm 0.00300 \\
2454399.68006 \pm 0.01700 \\
2454399.15242 \pm 0.00021 \\
2454398.07924 \pm 0.00024\end{array}$ & $\begin{array}{c}34.09600 \pm 0.00400 \\
2.73050 \pm 0.00074 \\
1.96475 \pm 0.00010 \\
1.87602 \pm 0.00001 \\
3.34726 \pm 0.00007 \\
36.76600 \pm 0.00100 \\
4.11230 \pm 0.00170 \\
1.68885 \pm 0.00017 \\
8.89880 \pm 0.00130 \\
0.95085 \pm 0.00021 \\
1.86559 \pm 0.00074 \\
1.99270 \pm 0.00040 \\
2.38102 \pm 0.00021 \\
1.24861 \pm 0.00024 \\
\end{array}$ & $\begin{array}{l}0.622 \\
0.640 \\
0.200 \\
2.444 \\
1.960 \\
4.700 \\
0.220 \\
0.240 \\
0.136 \\
0.083 \\
0.235 \\
0.130 \\
0.870 \\
0.684\end{array}$ & $\begin{array}{l}5.90 \\
3.86 \\
3.38 \\
2.93 \\
3.90 \\
3.50 \\
9.23 \\
3.57 \\
2.99 \\
4.80 \\
2.67 \\
2.81 \\
2.06 \\
2.79\end{array}$ \\
\hline $\begin{array}{l}102613782 \\
102674894 \\
102577194\end{array}$ & $\begin{array}{l}\text { E1 } 2960 \\
\text { E2 } 3389 \\
\text { E2 } 3612\end{array}$ & $\begin{array}{l}101.30976 \\
100.78138 \\
100.19875\end{array}$ & $\begin{array}{l}-0.46050 \\
-1.27590 \\
-1.43906\end{array}$ & $\begin{array}{c}\text { False alarms } \\
2454451.73910 \pm 0.01000 \\
2454459.32040 \pm 0.01000 \\
2454423.34000 \pm 0.11000\end{array}$ & $\begin{array}{c}13.03130 \pm 0.00330 \\
7.03490 \pm 0.00160 \\
38.24000 \pm 0.05000\end{array}$ & $\begin{array}{l}0.170 \\
0.076 \\
0.340\end{array}$ & $\begin{array}{l}9.50 \\
2.50 \\
8.56\end{array}$ \\
\hline $\begin{array}{l}102664130 \\
102613411\end{array}$ & $\begin{array}{l}\text { E2 } 0928 \\
\text { E2 } 5678\end{array}$ & $\begin{array}{l}100.72531 \\
100.43715\end{array}$ & $\begin{array}{l}-0.28241 \\
-0.55653\end{array}$ & $\begin{array}{l}\text { X-case candidates } \\
2454434.77350 \pm 0.01000 \\
2454412.56050 \pm 0.00500\end{array}$ & $\begin{array}{l}49.90000 \pm 0.10000 \\
18.76330 \pm 0.00050\end{array}$ & $\begin{array}{l}2.600 \\
4.330\end{array}$ & $\begin{array}{l}3.40 \\
8.00\end{array}$ \\
\hline
\end{tabular}

Notes. Transit depths are relative to the combined white flux. 
Table 6. CoRoT LRa01 planetary candidates: follow-up results.

\begin{tabular}{|c|c|c|c|c|c|c|c|c|}
\hline \multirow[t]{2}{*}{ Win ID } & \multirow{2}{*}{$\begin{array}{c}B \\
{[\mathrm{mag}]}\end{array}$} & \multirow{2}{*}{$\begin{array}{c}V \\
{[\mathrm{mag}]}\end{array}$} & \multirow{2}{*}{$\begin{array}{c}r^{\prime} \\
{[\mathrm{mag}]}\end{array}$} & \multirow{2}{*}{$\begin{array}{c}i^{\prime} \\
{[\mathrm{mag}]}\end{array}$} & \multirow{2}{*}{$\begin{array}{c}\text { Candidate } \\
\text { nature }^{a}\end{array}$} & \multicolumn{2}{|c|}{ Follow-up facilities } & \multirow[t]{2}{*}{ Comments } \\
\hline & & & & & & Photometry & Spectroscopy & \\
\hline \multicolumn{9}{|c|}{ Confirmed planets } \\
\hline E1 1031 & 14.656 & 14.018 & 13.760 & 13.405 & CoRoT-5b & IAC 80 & $\begin{array}{l}\text { AAOmega, } \\
\text { SOPHIE, } \\
\text { HARPS }\end{array}$ & Rauer et al. (2009) \\
\hline E2 0165 & 12.524 & 11.668 & 11.378 & 10.924 & CoRoT-7b & $\begin{array}{l}\text { CFHT, } \\
\text { CST/FASTCAM, } \\
\text { VLT/NACO, } \\
\text { IAC } 80\end{array}$ & $\begin{array}{l}\text { AAOmega, } \\
\text { FLAMES, } \\
\text { CRIRES, } \\
\text { HARPS, } \\
\text { UVES }\end{array}$ & $\begin{array}{l}\text { Léger et al. (2009); Queloz et al. (2009); } \\
\text { Hatzes et al. (2010); Bruntt et al. (2010); } \\
\text { Pont et al. (2011); Hatzes et al. (2011) }\end{array}$ \\
\hline E2 3459 & 16.343 & 15.515 & 15.211 & 14.685 & CoRoT-12b & IAC 80 & $\begin{array}{l}\text { HARPS, } \\
\text { HIRES }\end{array}$ & Gillon et al. (2010) \\
\hline E2 5277 & 16.946 & 16.090 & 15.726 & 15.175 & CoRoT-21b & IAC 80 & $\begin{array}{l}\text { HARPS, } \\
\text { HIRES }\end{array}$ & $\begin{array}{l}\text { IAC } 80 \text { confirms the transit is on target. } \\
\text { HARPS and HIRES RV data confirm a hot- } \\
\text { Jupiter planet with } M_{\mathrm{p}} \approx 2 M_{\text {Jup }} \text { transiting an } \\
\text { F8 IV star with } T_{\text {eff }} \approx 6100 \mathrm{~K} \text { and } \log g \approx 3.5 \\
\text { dex (Pätzold et al., in prep.). }\end{array}$ \\
\hline
\end{tabular}

$\begin{array}{lllllll}\text { E1 } 0544 & 14.021 & 13.392 & 13.132 & 12.750 \quad \text { CEB } & \begin{array}{l}\text { EulerCam, } \\ \text { IAC } 80\end{array} & \begin{array}{l}\text { AAOmega, } \\ \text { SOPHIE }\end{array}\end{array}$

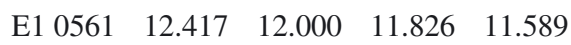

SB3

$\begin{array}{llllll}\text { E1 } 2890 & 17.189 & 15.732 & 15.121 & 14.215 & \text { CEB } \quad \text { IAC } 80\end{array}$

E1 $3666 \quad 16.172 \quad 15.470 \quad 15.133 \quad 14.668$

CEB CFHT, IAC 80

E1 $5015 \quad 17.167 \quad 16.168 \quad 15.743 \quad 15.087$

SB1 ESA-OGS

$\begin{array}{lllllll}\text { E1 } 4353 & 16.369 & 15.775 & 15.521 & 15.145 & \text { CEB } \quad \text { EulerCam, }\end{array}$
HARPS

AAOmega, SOPHIE, UVES

IAC80
Classified as F7 V by AAOmega. According to SOPHIE RV measurements, the star is a fast rotator and shows no RV variation down to a precision of $50 \mathrm{~m} \mathrm{~s}^{-1}$. EulerCam and IAC 80 photometry reveals that a $\sim 4$ mag fainter star, located $9^{\prime \prime}$ west of the main target, contaminates the lightcurve with deep eclipses $(D \approx 20 \%)$.

$\gamma$-Doradus variable star, classified as an A7IV/V star by AAOmega. SOPHIE finds a low-contrast single peak CCF with an RV variation of about $52 \mathrm{~km} \mathrm{~s}^{-1}$, in anti-phase with the CoRoT ephemeris. A single epoch UVES spectrum reveals an SB3 system.

IAC 80 observations find that a $\sim 3.2 \mathrm{mag}$ fainter star, located $\sim 12$ " southeast of the target, shows a $40 \%$ deep eclipse. Taking into account the light contamination inside the photometric mask, the observed variation is consistent with the CoRoT transit-like signal.

IAC 80 and CHFT observations find that a $\sim 0.5$ mag fainter star, located $\sim 8^{\prime \prime}$ west of the target, exhibits a $1.5 \%$ deep eclipse. Taking into account the light contamination inside the photometric mask, the observed variation is consistent with the CoRoT transit-like signal.

Transit duration of $10 \mathrm{~h}$ indicates an eclipsing binary at photometric detection level. ESA-OGS observes the transit on target. Two HARPS RV measurements confirm a binary system with low-mass companion $(K=$ $16.3 \mathrm{~km} \mathrm{~s}^{-1}$ if $e=0 ; M_{2} \approx 0.18 M_{\odot}$ if $\left.M_{1}=1 M_{\odot}\right)$.

EulerCam finds that the transit-like signal is caused by a $5 \%$ deep eclipse in a nearby contaminant star (CoRoT ID: 0102691690, $V=16.7 \mathrm{mag}$ ). CEB scenario confirmed by IAC 80. 
Table 6. continued.

\begin{tabular}{|c|c|c|c|c|c|c|c|c|}
\hline \multirow[t]{2}{*}{ Win ID } & \multirow{2}{*}{$\begin{array}{c}B \\
{[\mathrm{mag}]}\end{array}$} & \multirow{2}{*}{$\begin{array}{c}V \\
{[\mathrm{mag}]}\end{array}$} & \multirow{2}{*}{$\begin{array}{c}r^{\prime} \\
{[\mathrm{mag}]}\end{array}$} & \multirow{2}{*}{$\begin{array}{c}i^{\prime} \\
{[\mathrm{mag}]}\end{array}$} & \multirow{2}{*}{$\begin{array}{c}\text { Candidate } \\
\text { nature }^{a}\end{array}$} & \multicolumn{2}{|c|}{ Follow-up facilities } & \multirow[t]{2}{*}{ Comments } \\
\hline & & & & & & Photometry & Spectroscopy & \\
\hline E2 1123 & 15.934 & 14.622 & 13.983 & 13.185 & Blend & IAC 80, Wise & $\begin{array}{l}\text { HARPS, } \\
\text { CORALIE, } \\
\text { UVES }\end{array}$ & $\begin{array}{l}\text { Wise and IAC } 80 \text { observe the transit on target. } \\
\text { CORALIE, UVES, and HARPS show an active } \\
\text { K5 V star with no detected RV variation at a } \\
\text { level of about } 50 \mathrm{~m} \mathrm{~s}^{-1} \text {. UVES spectra reveal } 3 \\
\text { emission components in the core of the Ca II } \mathrm{H} \\
\& \mathrm{~K} \text { lines. The RVs of } 2 \text { components vary in } \\
\text { phase at twice the transit period and with a max- } \\
\text { imum velocity difference of } 67 \mathrm{~km} \mathrm{~s}^{-1} \text {. Blend } \\
\text { scenario: suspected hierarchical triple system } \\
\text { consisting of an active K-dwarf orbited by two } \\
\text { eclipsing active M-type stars. }\end{array}$ \\
\hline E2 1145 & 14.524 & 13.963 & 13.810 & 13.462 & SB1 & & $\begin{array}{l}\text { AAOmega, } \\
\text { SOPHIE }\end{array}$ & $\begin{array}{l}\text { Also known as IRa01 E1 1873. Classified as } \\
\text { A9IV/V by AAOmega. SOPHIE finds an RV } \\
\text { variation with a semi-aplitude } K=23.5 \mathrm{~km} \mathrm{~s}^{-1} \\
\text { (assuming } e=0 \text { ) in anti-phase with the CoRoT } \\
\text { ephemeris. }\end{array}$ \\
\hline E2 1897 & 15.407 & 14.721 & 14.476 & 14.098 & CEB & CFHT & & $\begin{array}{l}\text { Hints of a secondary eclipse are found in } \\
\text { the lightcurve. Contamination by an eclipsing } \\
\text { binary located } \sim 3^{\prime \prime} \text { northeast from the main } \\
\text { CoRoT target is found by CFHT photometry. }\end{array}$ \\
\hline E2 2249 & 15.019 & 13.876 & 13.423 & 12.813 & SB1 & & $\begin{array}{l}\text { AAOmega, } \\
\text { SOPHIE }\end{array}$ & $\begin{array}{l}\text { K0 III/IV star based on AA0mega spectra. } \\
\text { SOPHIE finds an RV semi-amplitude } K= \\
12.2 \mathrm{~km} \mathrm{~s}^{-1} \text { (assuming } e=0 \text { ) consistent with a } \\
\text { binary system with low-mass companion }\left(M_{2} \approx\right. \\
\left.0.17 M_{\odot} \text { if } M_{1}=1 M_{\odot}\right) \text {. }\end{array}$ \\
\hline E2 2481 & 14.885 & 13.958 & 13.598 & 13.059 & SB2 & & $\begin{array}{l}\text { AAOmega, } \\
\text { SOPHIE }\end{array}$ & $\begin{array}{l}\text { Also a mono-transit candidate in IRa01, known } \\
\text { as IRa01 E1 2046. AAOmega and SOPHIE } \\
\text { identify this candidate as an SB2 system. }\end{array}$ \\
\hline E2 2694 & 13.988 & 13.562 & 13.420 & 13.066 & B3Ve star & & $\begin{array}{l}\text { AAOmega, } \\
\text { SOPHIE, } \\
\text { HARPS }\end{array}$ & $\begin{array}{l}\text { SOPHIE and HARPS find no CCF, He I absorp- } \\
\text { tion lines, and strong emission Balmer lines. } \\
\text { AAOmega identifies the star as a B } 3 \text { Ve star. If } \\
\text { on target, the observed transit signal is caused } \\
\text { by an eclipsing star. }\end{array}$ \\
\hline E2 4129 & 16.575 & 15.705 & 15.395 & 14.775 & CEB & Eulercam, Wise & & $\begin{array}{l}\text { Wise observations are inconclusive. EulerCam } \\
\text { reveals a } 7 \% \text { drop in the flux of a contaminat- } \\
\text { ing star located } \sim 4.5^{\prime \prime} \text { north-northeast from the } \\
\text { CoRoT target and responsible for the detected } \\
\text { transit-like signal. }\end{array}$ \\
\hline E2 5084 & 16.703 & 15.948 & 15.685 & 15.172 & SB1 & & HARPS & $\begin{array}{l}\text { HARPS reveals an SB1 system with a } K= \\
37.2 \mathrm{~km} \mathrm{~s}^{-1} \mathrm{RV} \text { curve (assuming } e=0 \text { ) in anti- } \\
\text { phase with the CoRoT ephemeris. }\end{array}$ \\
\hline E2 5184 & 16.512 & 15.412 & 14.936 & 14.412 & Blend & CFHT & HARPS & $\begin{array}{l}\text { Also in IRa01 as IRa01 E1 4108. CFHT con- } \\
\text { firms the transit on target. HARPS spectra yield } \\
T_{\text {eff }}=5000 \pm 100 \mathrm{~K}, \log g=4.4 \pm 0.1 \mathrm{dex} \text {, } \\
{[\mathrm{M} / \mathrm{H}]=0.07 \pm 0.06 \mathrm{dex}, v \sin i=1.5 \pm} \\
0.5 \mathrm{~km} \mathrm{~s}^{-1} \text {, and SpT }=\mathrm{K} 0 \mathrm{~V} \text {. Strong HARPS } \\
\mathrm{CCF} \text { bisector-RV correlation identifies the can- } \\
\text { didate as a blend. }\end{array}$ \\
\hline E2 5747 & 16.855 & 16.158 & 15.868 & 15.382 & A-type star & & HARPS & $\begin{array}{l}\text { Also in IRa01 as IRa01 E1 4617. Transit dura- } \\
\text { tion ( } 14.13 \mathrm{~h} \text { ) and depth }(3.64 \%) \text { implies stellar } \\
\text { companion. HARPS finds no CCF. Target star } \\
\text { identified as a rapidly rotating A-type star. If on } \\
\text { target, the observed transit signal is caused by } \\
\text { an eclipsing star. }\end{array}$ \\
\hline E2 3739 & 16.283 & 15.546 & 15.261 & 14.736 & A-type star & EulerCam & HARPS & $\begin{array}{l}\text { Also a mono-transit candidate in IRa01, known } \\
\text { as IRa } 01 \mathrm{E} 14014 \text {. Transit depth ( } 2.93 \%) \text {, dura- } \\
\text { tion }(6.97 \mathrm{~h} \text { ), and shape indicate grazing stel- } \\
\text { lar eclipses. Transit observed by EulerCam } 0.15 \\
\text { days after the predicted time, but still consistent } \\
\text { with the transit event being on target. HARPS } \\
\text { spectroscopy shows no CCF and a rapidly ro- } \\
\text { tating A-type star. The observed transit signal is } \\
\text { caused by an eclipsing star. }\end{array}$ \\
\hline
\end{tabular}


Table 6. continued.

\begin{tabular}{|c|c|c|c|c|c|c|c|c|}
\hline \multirow[t]{2}{*}{ Win ID } & \multirow{2}{*}{$\begin{array}{c}B \\
{[\mathrm{mag}]}\end{array}$} & \multirow{2}{*}{$\begin{array}{c}V \\
{[\mathrm{mag}]}\end{array}$} & \multirow{2}{*}{$\begin{array}{c}r^{\prime} \\
{[\mathrm{mag}]}\end{array}$} & \multirow{2}{*}{$\begin{array}{c}i^{\prime} \\
{[\mathrm{mag}]}\end{array}$} & \multirow{2}{*}{$\begin{array}{l}\text { Candidate } \\
\text { nature }^{a}\end{array}$} & \multicolumn{2}{|c|}{ Follow-up facilities } & \multirow[t]{2}{*}{ Comments } \\
\hline & & & & & & Photometry & Spectroscopy & \\
\hline E2 5756 & 16.892 & 16.236 & 15.963 & 15.520 & A-type star & IAC 80 & HARPS & $\begin{array}{l}\text { Deep transit signal (2.72\%) with steep } \\
\text { ingress/egress. Transit on target according to } \\
\text { IAC } 80 \text { observations. HARPS finds no CCF } \\
\text { and a rapidly rotating A-type star. The ob- } \\
\text { served transit signal is caused by an eclipsing } \\
\text { star. }\end{array}$ \\
\hline
\end{tabular}

Unsettled good planetary candidates

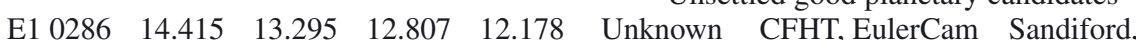

SOPHIE,

HARPS,

HIRES, FIES

$\begin{array}{llllll}\text { E1 } 2101 & 15.257 & 14.153 & 13.509 & 12.907 & \text { Unknown } \begin{array}{l}\text { CFHT, MONET- HARPS } \\ \text { North }\end{array}\end{array}$

$\begin{array}{llllll}\text { E1 2240 } & 15.859 & 15.221 & 14.914 & 14.475 & \text { Unknown } \\ & & & & & \\ \text { E1 3216 } & 16.470 & 15.694 & 15.331 & 14.856 & \text { Unknown } \\ \text { E1 3221 } & 16.350 & 15.584 & 15.201 & 14.685 & \text { Unknown }\end{array}$

$\begin{array}{llllll}\text { E1 } 4423 & 17.528 & 16.224 & 15.569 & 14.805 & \text { Unknown }\end{array}$

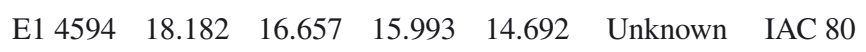

$\begin{array}{lllllll}\text { E1 } 4667 & 16.845 & 16.084 & 15.575 & 15.029 & \text { Unknown } & \text { IAC 80, Wise }\end{array}$ HARPS

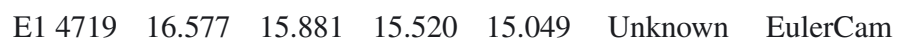

Sandiford, HARPS, SOPHIE, HIRES, and FIES RV measurements show that the G8/9 IV primary star $\left(T_{\text {eff }}=5250 \pm 80 \mathrm{~K}\right.$, $\log g=3.75 \pm 0.10 \mathrm{dex},[\mathrm{M} / \mathrm{H}]=-0.10 \pm$ $\left.0.05 \mathrm{dex}, v \sin i=3.0 \pm 1.0 \mathrm{~km} \mathrm{~s}^{-1}\right)$ belongs to a stellar binary system $(P=337.52 \pm$ 0.20 days, $e \approx 0.01, K=6.22 \pm 0.18 \mathrm{~km} \mathrm{~s}^{-1}$, yielding $M_{2} \approx 0.22 M_{\odot}$ if $M_{1}=1.10 M_{\odot}$ ). No RV variation in phase with the CoRoT ephemeris is found in the RV residuals down to $5 \mathrm{~m} \mathrm{~s}^{-1}$. CFHT and EulerCam photometric observations are not conclusive but might suggest a CEB scenario. The candidate is still under investigation.

The lightcurve shows a spot-induced $2 \%$ flux modulation with a period of $\sim 11$ days. The transit signal is only found in the CoRoT red channel because its depth is below the noise level in the green and blue channels. Combined measurements with CFHT and MONET-North exclude CEB. HARPS reveals $\mathrm{K} 6 \mathrm{~V}$ star with $T_{\text {eff }} \approx 4250 \mathrm{~K}$ and $\log g \approx 4.5$ dex. Six HARPS RV measurements show no significant sinusoidal variation down to a precision of $18 \mathrm{~m} \mathrm{~s}^{-1}$. The candidate is still under investigation.

Asymmetric transit shape. Owing to the low $S / N$ ratio this is not conclusive to rule out the candidate.

It shows shallow out-of-transit variations.

V-shaped transit. Pulsations with periods of 0.78 and 8.75 days, typical of a giant, are detected in the lightcurve, in disagreement with the A5 V classification reported in Exo-Dat.

V-shaped transit. The lightcurve is strongly affected by instrumental effects (jumps).

The $6.62 \mathrm{~h}$ transit duration suggests an evolved host star. IAC 80 excludes nearby CEBs. The transit is concluded to be on target.

Wise photometric observations are inconclusive because of bad weather. IAC 80 photometry excludes contamination by background eclipsing binaries. Two HARPS spectra unveil a G0V star and show an RV variation of $84 \mathrm{~m} \mathrm{~s}^{-1}$, which is comparable to the error bars $\left(\sim 70 \mathrm{~m} \mathrm{~s}^{-1}\right)$. The spectroscopic followup is still on-going.

V-shaped and asymmetric transit shape. EulerCam follow-up might have missed the transit owing to the timing errors $(2 \mathrm{~h})$ at the time of the observations (28 October 2010). Nevertheless, large photometric variations from nearby contaminant stars are probably not the cause for the observed transit event. 
Table 6. continued.

\begin{tabular}{|c|c|c|c|c|c|c|c|c|}
\hline \multirow[t]{2}{*}{ Win ID } & \multirow{2}{*}{$\begin{array}{c}B \\
{[\mathrm{mag}]}\end{array}$} & \multirow{2}{*}{$\begin{array}{c}V \\
{[\mathrm{mag}]}\end{array}$} & \multirow{2}{*}{$\begin{array}{c}r^{\prime} \\
{[\mathrm{mag}]}\end{array}$} & \multirow{2}{*}{$\begin{array}{c}i^{\prime} \\
{[\mathrm{mag}]}\end{array}$} & \multirow{2}{*}{$\begin{array}{c}\text { Candidate } \\
\text { nature }^{a}\end{array}$} & \multicolumn{2}{|c|}{ Follow-up facilities } & \multirow[t]{2}{*}{ Comments } \\
\hline & & & & & & Photometry & Spectroscopy & \\
\hline E1 4820 & 16.891 & 16.153 & 15.855 & 15.367 & Unknown & ESA-OGS & & $\begin{array}{l}\text { According to OGS-ESA observations, the } \\
\text { transit is on target. The lightcurve analy- } \\
\text { sis indicates possible out-of-transit variations } \\
\text { and depth differences between even and odd } \\
\text { transits. }\end{array}$ \\
\hline E1 5320 & 17.022 & 16.134 & 15.736 & 15.182 & Unknown & & & $\begin{array}{l}\text { Uncertain detection. The transit-like sig- } \\
\text { nal is only identified in the phase-folded } \\
\text { lightcurve. }\end{array}$ \\
\hline E1 5536 & 17.032 & 16.208 & 15.835 & 15.307 & Unknown & CFHT & & $\begin{array}{l}\text { Although CFHT observed a } 0.40 \pm 0.25 \% \\
\text { deep transit on target compatible with the } \\
\text { CoRoT signal, some of the nearby back- } \\
\text { ground stars could not be excluded as pos- } \\
\text { sible contaminants. Observations are consid- } \\
\text { ered inconclusive. }\end{array}$ \\
\hline E2 3156 & 17.211 & 15.757 & 15.127 & 14.271 & Unknown & IAC 80 & $\begin{array}{l}\text { HARPS, } \\
\text { HIRES }\end{array}$ & $\begin{array}{l}\text { The transit is only seen in the red CoRoT } \\
\text { channel because its depth is below the noise } \\
\text { level in the green and blue channels. It is } \\
\text { V-shaped and has a long duration }(\sim 2 \mathrm{~h}) \text { for a } \\
\text { planetary object. According to IAC } 80 \text { obser- } \\
\text { vations, background contaminants are prob- } \\
\text { ably excluded. There is a } 20-30 \% \text { chance } \\
\text { of missed transit owing to timing errors. } \\
\text { HARPS \& HIRES find no RV variation down } \\
\text { to a precision of } 10 \mathrm{~m} \mathrm{~s}^{-1} \text {. The candidate is } \\
\text { still under investigation. }\end{array}$ \\
\hline E2 3619 & 16.457 & 15.555 & 15.167 & 14.608 & Unknown & & AAOmega & $\begin{array}{l}\text { Also in IRa01 as IRa01 E1 2060. G8 V } \\
\text { star according to Exo-Dat. Classified as a } \\
\text { G0 IV/V star by low-resolution AAOmega } \\
\text { observations. CoRoT photometry shows } \\
\text { multi-periodic variations with frequency } \\
\text { spacing consistent with a giant. True spectral } \\
\text { type unclear. }\end{array}$ \\
\hline E2 4519 & 16.316 & 15.748 & 15.510 & 15.134 & Unknown & $\begin{array}{l}\text { EulerCam, } \\
\text { IAC } 80\end{array}$ & & $\begin{array}{l}\text { EulerCam and IAC } 80 \text { observations show no } \\
\text { photometric variations in any of the nearby } \\
\text { stars. However, the transit might have been } \\
\text { missed owing to the timing errors ( } 1.5 \mathrm{~h} \text { ) } \\
\text { at the time of the observations (Nov.-Dec. } \\
\text { 2010). Still, the transit-like signal is consid- } \\
\text { ered to arise from the target. }\end{array}$ \\
\hline
\end{tabular}

$\begin{array}{lllll}\text { E1 } 2970 & 15.057 & 14.487 & 14.263 & 13.837\end{array}$ Unsettled low-priority planetary candidates

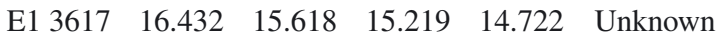

E1 $3674 \quad 15.785 \quad 15.324 \quad 15.146 \quad 14.842 \quad$ Unknown

$\begin{array}{llllll}\text { E1 } & 4272 & 16.750 & 15.868 & 15.506 & 14.889\end{array}$ Unknown ESA-OGS

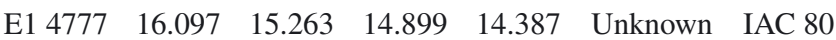

V-shaped transit signal detected only in the red channel. No significant detection in the blue and green channels. Hints of secondary eclipse in the red lightcurve. Suspected CEB. Several frequencies coherent with the transit period point to activities induced by a massive companion. Hints of secondary eclipses in the lightcurve. Suspected eclipsing binary. V-shaped transit with long duration $(3.38 \mathrm{~h})$ seen only in the red channel. No significant detection in the blue and green lightcurve. Suspected CEB.

ESA-OGS confirms the transit signal to be on target. Out-of-transit variations detected in the lightcurve. Suspected eclipsing binary. V-shaped transit with long duration (3.90 h) and depth differences by more than $1 \sigma$ in the three color channels. No transit detected by IAC 80 , possibly owing to underestimated transit timing error at the time of the observations (February 2011).

V-shaped signal with significant $(12 \sigma)$ depth difference between even and odd transits. Suspected eclipsing binary. 
Table 6. continued.

\begin{tabular}{|c|c|c|c|c|c|c|c|c|}
\hline \multirow[t]{2}{*}{ Win ID } & \multirow{2}{*}{$\begin{array}{c}B \\
{[\mathrm{mag}]}\end{array}$} & \multirow{2}{*}{$\begin{array}{c}V \\
{[\mathrm{mag}]}\end{array}$} & \multirow{2}{*}{$\begin{array}{c}r^{\prime} \\
{[\mathrm{mag}]}\end{array}$} & \multirow{2}{*}{$\begin{array}{c}i^{\prime} \\
{[\mathrm{mag}]}\end{array}$} & \multirow{2}{*}{$\begin{array}{l}\text { Candidate } \\
\text { nature }^{a}\end{array}$} & \multicolumn{2}{|c|}{ Follow-up facilities } & \multirow[t]{2}{*}{ Comments } \\
\hline & & & & & & Photometry & Spectroscopy & \\
\hline E1 5450 & 17.537 & 16.382 & 15.878 & 15.163 & Unknown & & & $\begin{array}{l}\text { Asymmetric transit shape. Duration too long } \\
(9.23 \mathrm{~h}) \text { to be consistent with a transiting } \\
\text { planet. }\end{array}$ \\
\hline E2 2185 & 15.830 & 15.078 & 14.772 & 14.299 & Unknown & & & $\begin{array}{l}\text { Also in IRa01 as IRa01 E1 } 1319 \text {. V-shaped } \\
\text { long transit signal }(3.57 \mathrm{~h}) \text {. Faint secondary } \\
\text { eclipse at phase } 0.5 \text { and depth differences } \\
\text { between even and odd transits suggest an } \\
\text { eclipsing binary. }\end{array}$ \\
\hline E2 2597 & 15.286 & 14.165 & 13.753 & 12.980 & Unknown & & $\begin{array}{l}\text { AAOmega, } \\
\text { FLAMES }\end{array}$ & $\begin{array}{l}\text { The signal is only present in the blue chan- } \\
\text { nel. No significant detection in the green } \\
\text { and red lightcurve. Classified as a G6 III/IV } \\
\text { star by AAOmega. FLAMES yields } T_{\text {eff }}= \\
4991 \pm 140 \mathrm{~K}, \log g=3.24 \pm 0.30 \text { dex, } \\
{[\mathrm{M} / \mathrm{H}]=-0.29 \pm 0.15 \mathrm{dex} \text {, and } v \sin i=} \\
4.8 \pm 2.0 \mathrm{~km} \mathrm{~s}^{-1} \text {. Suspected CEB. }\end{array}$ \\
\hline E2 2627 & 15.872 & 15.127 & 14.848 & 14.358 & Unknown & & AAOmega & $\begin{array}{l}\text { V-shaped transit signal found only in the } \\
\text { CoRoT blue channel. No significant detec- } \\
\text { tion in the green and red channels. Classified } \\
\text { as an F4 V star by AAOmega. Suspected } \\
\text { CEB. }\end{array}$ \\
\hline E2 3157 & 15.990 & 14.858 & 14.351 & 13.715 & Unknown & & & $\begin{array}{l}\text { V-shaped signal found only in the red chan- } \\
\text { nel with significant }(5 \sigma) \text { depth differences } \\
\text { between even and odd transits. Suspected } \\
\text { CEB. }\end{array}$ \\
\hline E2 4494 & 17.225 & 16.069 & 15.561 & 14.795 & Unknown & & & $\begin{array}{l}\text { Asymmetric transit signal with long duration } \\
(2.81 \mathrm{~h}) \text {. }\end{array}$ \\
\hline E2 4910 & 16.240 & 15.357 & 14.977 & 14.447 & Unknown & IAC 80 & AAOmega & $\begin{array}{l}\text { Also in IRa01 as IRa01 E1 } 1531 \text {. Classified } \\
\text { as an F7/8 V star according to AAOmega. } \\
\text { IAC } 80 \text { observes the transit on target. } \\
\text { Secondary eclipses found in the lightcurve. } \\
\text { Suspected eclipsing binary. }\end{array}$ \\
\hline E2 5194 & 17.126 & 16.093 & 15.667 & 15.154 & Unknown & & & $\begin{array}{l}\text { Transit duration too long }(2.79 \mathrm{~h}) \text { for a plane- } \\
\text { tary companion. Suspected eclipsing binary. }\end{array}$ \\
\hline \multicolumn{9}{|c|}{ False alarms } \\
\hline E1 2960 & 15.144 & 14.400 & 14.061 & 13.625 & Unknown & & & $\begin{array}{l}\text { Transit-like signal detected only in the } \\
\text { CoRoT red channel. Instrumental effects } \\
\text { (jumps) in the lightcurve might mimic the } \\
\text { transit-like signal. }\end{array}$ \\
\hline E2 3389 & 16.948 & 15.649 & 15.087 & 14.127 & Unknown & & & $\begin{array}{l}\text { Instrumental effects (jumps) in the lightcurve } \\
\text { might mimic the transit-like signal. }\end{array}$ \\
\hline E2 3612 & 17.369 & 16.013 & 15.482 & 14.541 & Unknown & & & $\begin{array}{l}\text { Asymmetric transit shape. Instrumental ef- } \\
\text { fects (jumps) in the lightcurve might mimic } \\
\text { the transit-like signal. }\end{array}$ \\
\hline \multicolumn{9}{|c|}{$\mathrm{X}$-case candidates } \\
\hline E2 0928 & 16.035 & 15.618 & 15.476 & 15.220 & Unknown & & & $\begin{array}{l}\text { Transit signal too deep }(2.60 \%) \text { for a plane- } \\
\text { tary candidate if spectral type is A5 IV (Exo- } \\
\text { Dat). It needs spectral type confirmation. }\end{array}$ \\
\hline E2 5678 & 16.505 & 15.909 & 15.617 & 15.257 & Unknown & & & $\begin{array}{l}\text { Transit signal too deep }(4.33 \%) \text { for a plane- } \\
\text { tary candidate if spectral type is F } 8 \text { (Exo- } \\
\text { Dat). It needs spectral type confirmation. }\end{array}$ \\
\hline
\end{tabular}

Notes. ${ }^{(a)}$ The following abbreviations are used. CEB: Spatially resolved contaminating eclipsing binary system whose light is diluted by the main CoRoT target. SB1: Binary system with one spectroscopically visible stellar component. SB2: Binary system with two spectroscopically visible stellar components. SB3: Triple system with three spectroscopically visible stellar components. Blend: spatially unresolved eclipsing binary system whose light is diluted by the main CoRoT target. 


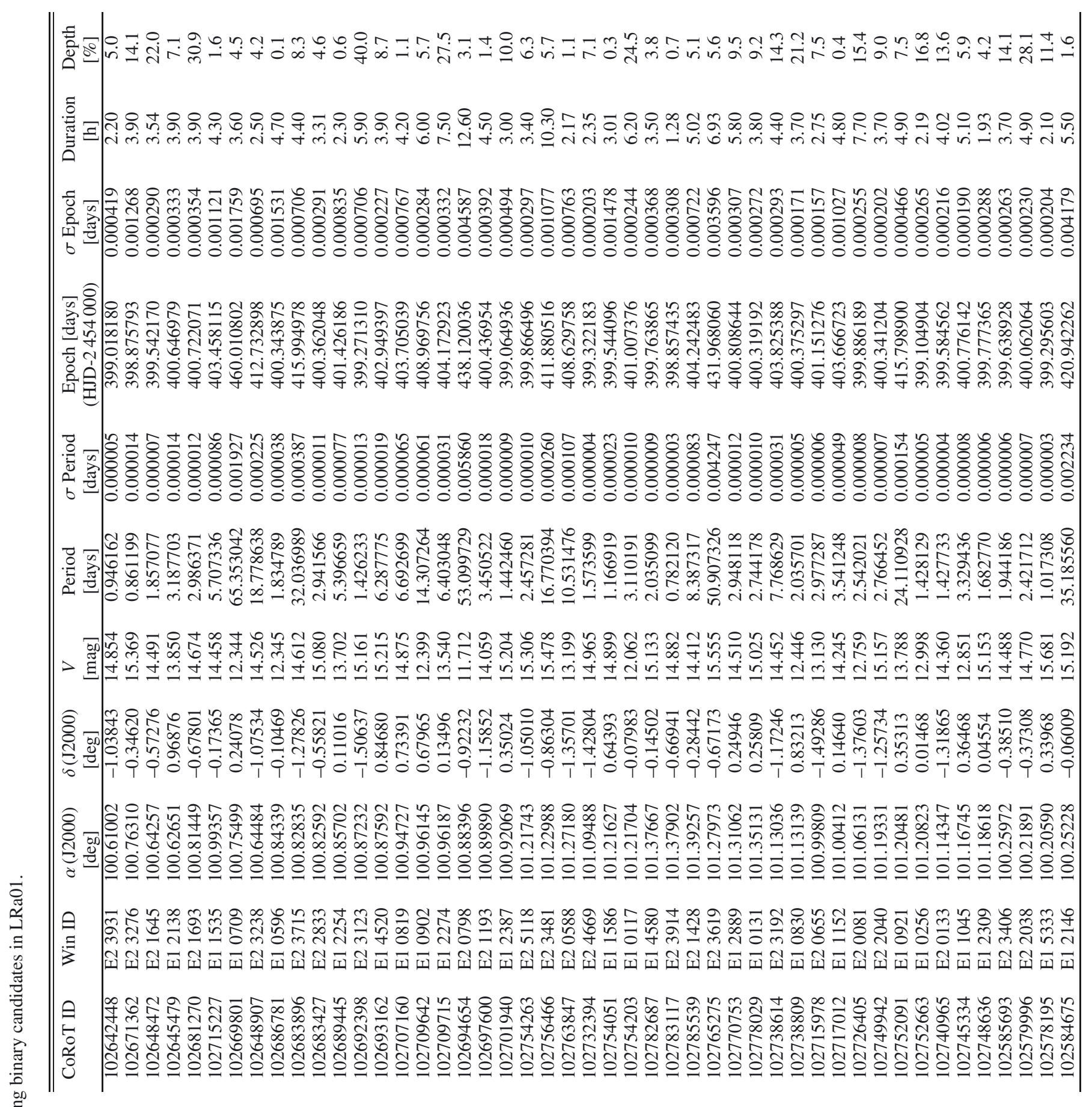




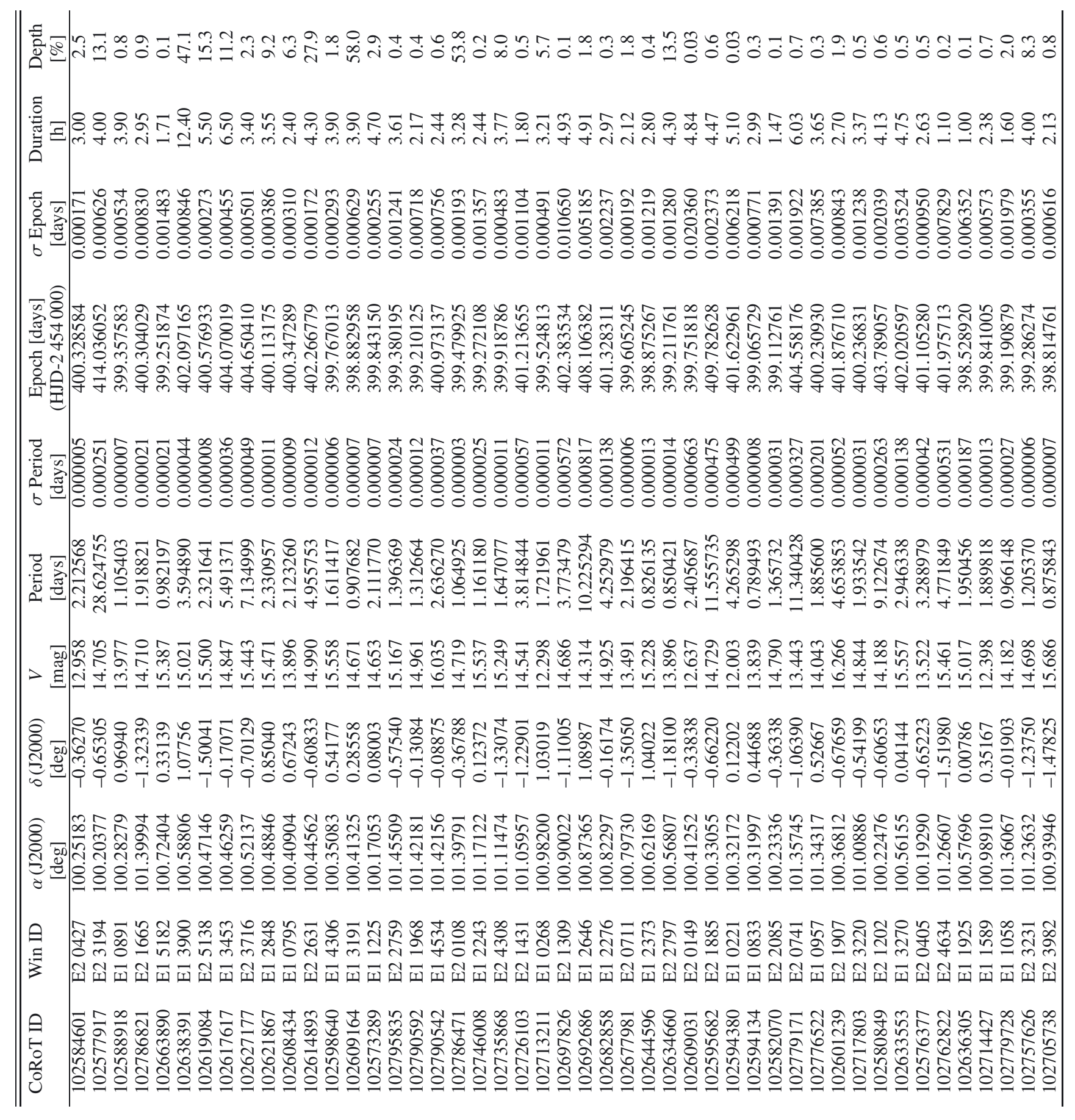




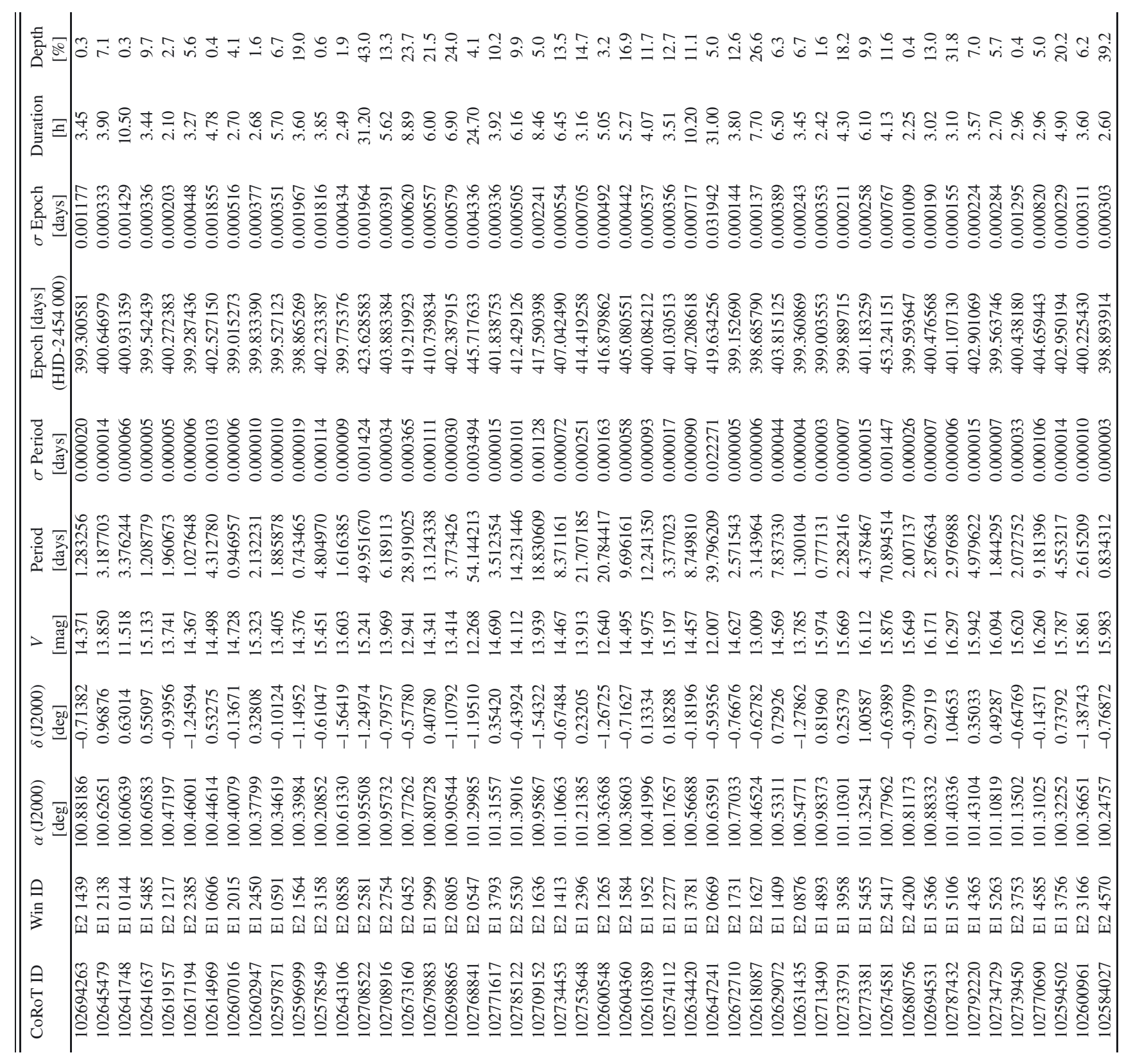


